Zou, H.L. Abdel-Malek, K. and Wang, J., (1997), "Design Propagation in Mechanical Systems: Kinematic Analysis," ASME Journal of Mechanical Design, Vol. 119, No. 3, pp. 338-345.

\title{
Design Propagation in Mechanical Systems: Kinematic Analysis
}

\author{
H. Zou \\ K. A. Abdel-Malek \\ J. Y. Wang \\ Center for Computer Aided Design (CCAD) and \\ Department of Mechanical Engineering \\ The University of Iowa \\ Iowa City, IA 52242
}

\begin{abstract}
A broadly applicable formulation for investigating design propagations in mechanisms is developed and illustrated. Analytical criteria in terms of the variations of joint position vectors and orientation matrices for planar and spatial mechanisms are presented. Mechanisms are represented using graph theory and closed loops are converted to a tree-like structure by cutting joints and introducing new constraints. The Jacobian matrix in Cartesian space is then transformed to Joint coordinates space. Two cases are considered: a pair of bodies remain connected by one joint after cutting additional joints and a pair of bodies are disconnected after cutting joints. Using this method, a designer has the ability to study the propagated effect of changing a design variable on the design. The presented formulation is validated through a numerical example of a McPherson strut suspension system. The system is analyzed and an assembled configuration is computed after a change in design.
\end{abstract}

\section{Introduction}

Effects of design propagations due to change in design variables is a relatively new field of study that has emerged as a result of more sophisticated computer-aided-engineering software. The field of concurrent engineering has seen many advances in recent years such as those exhibited in the following computer programs: DESIGNS (Silverstein et al. 1990), DAISIE (Alder et al. 1989), SATISFICER (Gopalakrishnan et al. 1990), NEXT-CUT (Cutkosky and Tenebaum 1990), CONCEPT MODELER (Serrano and Gossard 1988), STRUCTURAL DESIGN EVALUATOR (Fisher and Nguyen 1989), GALILEO3 (Bowen and Bahler 1992), STAURN (Fohn, et al. 1994), XCODOMAS (Burke et al. 1994), FDL (Imamura 1994), and FRODO (Kolb and Bailey 1993).

The concept of graph theory applied to kinematic and dynamic analysis was used by Wittenburg (1977) to simplify the representation of mechanisms using a computer. A mechanism is modeled into a spanning tree where a body is defined as a node and a kinematic joint is defined as an edge. If there are no closed loops in the system graph, the system is said to have a tree structure. If a graph is not a tree, an edge is cut in each independent closed loop to form a tree structure, called a spanning tree (Tsai 1989, Bae and Haug 1987, and Bae et al. 
1988). This type of formulation was implemented into parallel computational techniques for real-time simulation (Hwang et al. 1988 and Hwang and Haug 1988). Cut-joints are used to handle closed-loop systems to form a spanning tree that has no closed loops. Joints that are cut in the topology analysis process are replaced by a set of constraint equations.

Constraints between solid bodies are often characterized by conditions of orthogonality or parallelism of pairs of such vectors. The original work for deriving a library of possible constraints was presented by Haug (1989), and was implemented into a commercial dynamics analysis software called DADS (Cadsi 1995). The study of the derivatives of the mechanism's performance with respect to a design variable is called design sensitivity analysis (Tak and Kim 1990). The work by Haug and colleagues is further expanded here to include the joint position vectors that characterize a joint location with respect to a local body reference frame and orientation matrices that define joint orientation. These parameters are included as design variables to study design propagations. Derivatives of these vectors with respect to design parameters are computed in order to calculate the change in design using the generalized inverse method.

First, kinematic constraints due to cutting joints will be developed and differentiated with respect to design variables. Secondly, the Jacobian matrix is transformed from Cartesian coordinate space to Joint coordinate space. Cutting joints will result in two possible cases: (a) a pair of bodies remain connected by one joint or (b) a pair of bodies are disconnected. The two cases will be addressed. Finally, a numerical example will be used to illustrate the formulation.

The analytical formulation developed in this paper and validated through numerical examples is ultimately intended for implementation into computer-aided-engineering environments to increase the automation of mechanical design. It can also be used to study different scenarios of a particular design. The ultimate goal is to extend this work to include the propagation of dynamics in mechanisms and machines.

\section{Cut-Joint Constraint Formulation}

Cut-joint methods (Wittenburg 1977 and Haug 1989) are used to handle closed-loop systems to form a spanning tree that has no closed loops. Joints that are cut in the topology analysis process are replaced by a set of constraint equations. Partial derivatives of basic constraints with respect to design variables are derived in Cartesian coordinates. Figure 1 depicts a pair of solid bodies denoted by body $i$ and body $j$. The symbols $O_{i j}^{\prime \prime}$ and $O_{j i}^{\prime \prime}$ denote the origins of joint reference frames embedded in bodies $i$ and body $j$, respectively. The global reference frame is $x y z$, the body reference frame is $x^{\prime} y^{\prime} z^{\prime}$, and the joint reference frame is $x^{\prime \prime} y^{\prime \prime} z^{\prime \prime}$.

Fig. 1 Kinematic notation for vectors

In this section, four types of constraints are considered. The combination of these basic constraints can be used to define the kinematic joints between two links. The formulation in this 
section is aimed at determining partial derivatives of these basic constraints and extending the work to include the variations of joint position vectors and orientation matrices.

2.1 Dot-1 Constraint. A condition that a pair of body-fixed nonzero vectors $\mathbf{a}_{i}$ on body $i$ and $\mathbf{a}_{j}$ on body $j$ are orthogonal is that their scalar product be zero, such that

$$
\Phi^{d 1}\left(\mathbf{a}_{i}, \mathbf{a}_{j}\right) \equiv \mathbf{a}_{i}^{T} \mathbf{a}_{j}=0
$$

where the superscript $d l$ denotes the scalar product (dot product). The symbol $\Phi$ is used to indicate a constraint. The vector $\mathbf{a}_{i}$ can be written as $\mathbf{a}_{i}=\mathbf{A}_{i} \mathbf{C}_{i j} \mathbf{a}_{i}^{\prime \prime}$, where the _" symbol indicates vectors resolved with respect to the joint reference frames, $\mathbf{A}$ is the direction cosine matrix from the body reference frame $x^{\prime} y^{\prime} z^{\prime}$ to the global reference frame $x y z, \mathbf{C}$ is the transformation matrix from the joint reference frame $x^{\prime \prime} y^{\prime \prime} z^{\prime \prime}$ to the body reference frame $x^{\prime} y^{\prime} z^{\prime}$. The variation of $\mathbf{a}_{i}$ keeping $\mathbf{C}_{i j}$ as a design variable, can be written as

$$
\delta \mathbf{a}_{i}=\delta \mathbf{A}_{i} \mathbf{C}_{i j} \mathbf{a}_{i}^{\prime \prime}+\mathbf{A}_{i} \delta \mathbf{C}_{i j} \mathbf{a}_{i}^{\prime \prime}
$$

Define a global vector $\delta \pi$, called the virtual rotation of the transformation matrix $\mathbf{A}$ from the body reference frame $x^{\prime} y^{\prime} z^{\prime}$ to the global reference frame $x y z$. Also define $\delta \tilde{\pi}=\delta \mathbf{A} \mathbf{A}^{T}$, as a skew symmetric matrix (Haug 1989), where the tilde operator $(\sim)$ on a vector $\mathbf{a}=\left[\begin{array}{lll}a_{x} & a_{y} & a_{z}\end{array}\right]^{T}$, generates the following skew symmetric matrix.

$$
\widetilde{\mathbf{a}}=\left[\begin{array}{ccc}
0 & -a_{z} & a_{y} \\
a_{z} & 0 & -a_{x} \\
-a_{y} & a_{x} & 0
\end{array}\right]
$$

In order to study the variations of body orientations, define the vector $\delta \xi_{i j}$ as a local vector, called the virtual rotation of the transformation matrix $\mathbf{C}_{i j}$ from the joint reference frame $x^{\prime \prime} y^{\prime \prime} z^{\prime \prime}$ to the body reference frame $x^{\prime} y^{\prime} z^{\prime}$. Also define $\delta \tilde{\xi}_{i j}$ as a skew symmetric matrix such that $\delta \tilde{\xi}_{i j}^{\prime}=\delta \mathbf{C}_{i j} \mathbf{C}_{i j}^{T}$. Therefore, the variation of $\mathbf{A}$ can be written as $\delta \mathbf{A}=\mathbf{A} \delta \tilde{\pi}^{\prime}=\delta \tilde{\pi} \mathbf{A}$ and the variation of $\mathbf{C}$ can be written as $\delta \mathbf{C}_{i j}=\delta \tilde{\xi}_{i j}^{\prime} \mathbf{C}_{i j}$.

Substituting for $\delta \mathbf{A}, \delta \mathbf{C}_{i j}$ and the following property $\mathbf{A} \delta \tilde{\xi}_{i j}^{\prime}=\delta \tilde{\xi}_{i j}^{\prime} \mathbf{A}$ into Eq. (2) yields

$$
\delta \mathbf{a}_{i}=\delta \tilde{\pi}_{i} \mathbf{A}_{i} \mathbf{C}_{i j} \mathbf{a}_{i}^{\prime \prime}+\mathbf{A}_{i} \delta \tilde{\xi}_{i j}^{\prime} \mathbf{C}_{i j} \mathbf{a}^{\prime \prime}
$$

Replacing the expression for $\mathbf{a}_{i}$ into Eq. (4) and using the relationship $\tilde{\mathbf{a}} \mathbf{b}=-\tilde{\mathbf{b}} \mathbf{a}$ yields

$$
\delta \mathbf{a}_{i}=-\widetilde{\mathbf{a}}_{i} \delta \pi_{i}-\widetilde{\mathbf{a}}_{i} \delta \xi_{i j}^{\prime}
$$

The variation of the dot-1 constraint function of Eq. (1) can be written as

$$
\delta \Phi^{d 1}=\delta\left(\mathbf{a}_{i}^{T} \mathbf{a}_{j}\right)=\mathbf{a}_{j}^{T} \delta \mathbf{a}_{i}+\mathbf{a}_{i}^{T} \delta \mathbf{a}_{j}
$$

Substituting for $\delta \mathbf{a}_{i}$ and $\delta \mathbf{a}_{j}$ into Eq. (6) yields

$$
\delta \Phi^{d 1}=-\mathbf{a}_{j}^{T} \widetilde{\mathbf{a}}_{i} \delta \pi_{i}-\mathbf{a}_{j}^{T} \widetilde{\mathbf{a}}_{i} \delta \xi_{i j}^{\prime}-\mathbf{a}_{i}^{T} \widetilde{\mathbf{a}}_{j} \delta \pi_{j}-\mathbf{a}_{i}^{T} \widetilde{\mathbf{a}}_{j} \delta \xi_{j i}^{\prime}
$$

2.2 Dot-2 Constraint. Orthogonality of the body-fixed vector $\mathbf{a}_{i}$ and the vector $\mathbf{d}_{i j}$ can be written as: 


$$
\delta \Phi^{d 2}\left(\mathbf{a}_{i}, \mathbf{d}_{i j}\right)=\mathbf{a}_{i}^{T} \mathbf{d}_{i j}=0
$$

Referring to Fig. 1, the vector $\mathbf{d}_{i j}$ between two bodies can be written as

$$
\mathbf{d}_{i j}=\mathbf{r}_{j}+\mathbf{s}_{j i}-\mathbf{r}_{i}-\mathbf{s}_{i j}
$$

where $\mathbf{s}_{i j}$ are the joint position vectors that define the basic dimensions of a body. The variations of Eq. (9) is

$$
\delta \mathbf{d}_{i j}=\delta \mathbf{r}_{j}+\delta \mathbf{s}_{j i}-\delta \mathbf{r}_{i}-\delta \mathbf{s}_{i j}
$$

Note that $\mathbf{s}_{i j}$, throughout this discussion, is not considered as a constant and therefore its variation will be kept as a variable. The vectors $\mathbf{s}_{i j}$ and $\mathbf{s}_{j i}$ can be written as $\mathbf{s}_{i j}=\mathbf{A}_{i} \mathbf{s}_{i j} \quad$ and $\mathbf{S}_{j i}=\mathbf{A}_{j} \mathbf{S}_{j i}^{\prime}$. The variation of the joint position vectors can be written as

$$
\delta \mathbf{s}_{i j}=-\widetilde{\mathbf{s}}_{i j} \delta \boldsymbol{\pi}_{i}+\mathbf{A}_{i} \delta \mathbf{s}_{i j}^{\prime}
$$

Substituting for $\delta \mathbf{s}_{i j}$ and $\delta \mathbf{s}_{j i}$ into Eq. (10) yields

$$
\delta \mathbf{d}_{i j}=\delta \mathbf{r}_{j}-\delta \mathbf{r}_{i}-\widetilde{\mathbf{s}}_{j i} \delta \pi_{j}+\mathbf{A}_{j} \delta \mathbf{s}_{j i}^{\prime}+\widetilde{\mathbf{s}}_{i j} \delta \pi_{i}-\mathbf{A}_{i} \delta \mathbf{s}_{j i}^{\prime}
$$

The differential of the dot-2 constraint function of Eq. (8) can be written as

$$
\delta \Phi^{d 2}=\delta\left(\mathbf{a}_{i}^{T} \mathbf{d}_{i j}\right)=\mathbf{d}_{i j}^{T} \delta \mathbf{a}_{i}+\mathbf{a}_{i}^{T} \delta \mathbf{d}_{i j}
$$

Performing the derivation and substituting for $\delta \mathbf{d}_{i j}$ and $\delta \mathbf{a}_{i}$ yields

$$
\begin{array}{r}
\delta \Phi^{d 2}=\mathbf{a}_{i}^{T} \delta \mathbf{r}_{j}-\mathbf{a}_{i}^{T} \delta \mathbf{r}_{i}+\left(\mathbf{a}_{i}^{T} \widetilde{\mathbf{s}}_{i j}-\mathbf{d}_{i j}^{T} \widetilde{\mathbf{a}}_{i}\right) \delta \pi_{i}-\mathbf{a}_{i}^{T} \widetilde{\mathbf{s}}_{j i} \delta \boldsymbol{\pi}_{j} \\
\mathbf{a}_{i}^{T} \mathbf{A}_{j} \delta \mathbf{s}_{j i}^{\prime}-\mathbf{a}_{i}^{T} \mathbf{A}_{i} \delta \mathbf{s}_{i j}^{\prime}-\mathbf{d}_{i j}^{T} \widetilde{\mathbf{a}}_{i} \delta \xi_{i j}^{\prime}
\end{array}
$$

2.3 Spherical Constraint. A spherical joint is a type of constraint that requires a pair of points on two bodies to coincide. A necessary and sufficient condition for $O_{i j}^{\prime \prime}$ and $O_{j i}^{\prime \prime}$, to coincide is that $\mathbf{d}_{i j}=\mathbf{0}$; i.e.,

$$
\Phi^{s}\left(O_{i j}^{\prime \prime}, O_{j i}^{\prime \prime}\right)=\mathbf{r}_{j}+\mathbf{s}_{j i}-\mathbf{r}_{i}-\mathbf{s}_{i j}=\mathbf{0}
$$

Taking the variation of Eq. (15) and substituting for $\delta \mathbf{s}_{i j}$ and $\delta \mathbf{s}_{j i}$ yields

$$
\delta \Phi^{s}=\delta \mathbf{r}_{j}-\delta \mathbf{r}_{i}-\widetilde{\mathbf{s}}_{j i} \delta \pi_{j}+\mathbf{A}_{j} \delta \mathbf{s}_{j i}^{\prime}+\widetilde{\mathbf{s}}_{i j} \delta \pi_{i}-\mathbf{A}_{i} \delta \mathbf{s}_{j i}^{\prime}
$$

2.4 Distance Constraint. A distance constraint requires a specified distance between a pair of points. A necessary and sufficient condition that the distance between $O_{i j}^{\prime \prime}$ and $O_{j i}^{\prime \prime}$, shown in Fig. 1, be equal to $\ell \neq 0$ is that

$$
\Phi^{d i s}\left(O_{i j}^{\prime \prime}, O_{j i}^{\prime \prime}, \ell\right)=\mathbf{d}_{i j}^{T} \mathbf{d}_{i j}-\ell^{2}=0
$$

The differential of the distance constraint of Eq. (17) is

$$
\delta \Phi^{d i s}=2 \mathbf{d}_{i j}^{T} \delta \mathbf{d}_{i j}=2 \mathbf{d}_{i j}^{T}\left[\delta \mathbf{r}_{j}-\delta \mathbf{r}_{i}-\widetilde{\mathbf{s}}_{j i} \delta \pi_{j}+\mathbf{A}_{j} \delta \mathbf{s}_{j i}^{\prime}+\widetilde{\mathbf{s}}_{i j} \delta \pi_{i}-\mathbf{A}_{i} \delta \mathbf{s}_{i j}^{\prime}\right]
$$

2.5 General Formulation of Basic Constraints. All constraint equations can be written in the form

$$
\Phi\left(\mathbf{r}_{i}, \mathbf{A}_{i}, \mathbf{s}_{i j}, \mathbf{C}_{i j}, \mathbf{r}_{j}, \mathbf{A}_{j}, \mathbf{C}_{j i}, \mathbf{s}_{j i}\right)=\mathbf{0}
$$

The differential of both sides of Eq. (19), noting that $\mathbf{s}$ and $\mathbf{C}$ are variables, yields

$$
\begin{gathered}
\delta \Phi=\Phi_{\mathbf{r}_{i}} \delta \mathbf{r}_{i}+\Phi_{\pi_{i}} \delta \pi_{i}+\Phi_{\mathbf{r}_{j}} \delta \mathbf{r}_{j}+\Phi_{\pi_{j}} \delta \pi_{j}+\Phi_{\mathbf{s}_{i j}} \delta \mathbf{s}_{i j}^{\prime}+\Phi_{\mathbf{s}_{j i}} \delta \mathbf{s}_{j i}^{\prime} \\
\Phi_{\xi_{i j}} \delta \xi_{i j}^{\prime}+\Phi_{\xi_{j i}} \delta \xi_{j i}^{\prime}=\mathbf{0}
\end{gathered}
$$


In order to minimize the size of the system to be solved, a relative joint coordinate system is adopted. In the following section, the Jacobian matrix in Cartesian coordinate space denoted by $\Phi_{\hat{\mathbf{z}}}$ is transformed into joint coordinate space denoted by $\Phi_{\mathbf{q}}$ where $\Phi_{\mathbf{q}}=\left[\partial \Phi_{i} / \partial q_{j}\right]$ and $\mathbf{q}$ is the vector of relative joint coordinates. In order to convert a closed loop system to a tree structure, a joint is cut and an additional constraint is introduced as shown in Figure (1).

The general form of Eq. (20) can be written in a linearized form as

$$
\delta \Phi=\Phi_{\mathbf{z}_{i}} \delta \mathbf{z}_{i}+\Phi_{\mathbf{z}_{j}} \delta \mathbf{z}_{j}+\Phi \xi^{i j} \delta \xi^{i j}+\Phi_{\mathbf{s}^{i j}} \delta \mathbf{s}^{i j}
$$

where the virtual displacement for bodies $i$ and $j$ are $\delta \mathbf{z}_{\mathrm{i}}=\left[\begin{array}{ll}\delta \mathbf{r}_{i} & \delta \pi_{i}\end{array}\right]^{T}$ and $\quad \delta \mathbf{z}_{j}=\left[\begin{array}{ll}\delta \mathbf{r}_{j} & \delta \pi_{j}\end{array}\right]^{T}$. and the virtual joint position vector is $\delta \mathbf{s}^{i j}=\left[\begin{array}{ll}\delta \mathbf{s}_{i j}^{\prime} & \delta \mathbf{s}_{j i}^{\prime}\end{array}\right]^{T}$ and the virtual joint orientation vector is $\delta \xi^{i j}=\left[\begin{array}{ll}\delta \xi_{i j}^{\prime} & \delta \xi_{j i}^{\prime}\end{array}\right]^{T}$. The coefficients of virtual displacement are defined as $\Phi_{\mathbf{z}_{i}}=\left[\begin{array}{ll}\Phi_{\mathbf{r}_{i}} & \Phi_{\pi_{i}}\end{array}\right]$ and $\Phi_{\mathbf{z}_{j}}=\left[\begin{array}{ll}\Phi_{\mathbf{r}_{j}} & \Phi_{\pi_{j}}\end{array}\right]$. The coefficients of linkage length and rotation are defined as $\Phi_{\mathbf{s}^{i j}}=\left[\begin{array}{ll}\Phi_{\mathbf{s}_{i j}^{\prime}} & \Phi_{\mathbf{s}_{j i}^{\prime}}\end{array}\right]$ and $\Phi_{\xi^{i j}}=\left[\begin{array}{ll}\Phi_{\xi_{i j}} & \Phi_{\xi_{j i}}\end{array}\right]$.

\section{Transformation of Jacobian Matrix from Cartesian Space to Joint Coordinate Space}

3.1 Derivation of the Virtual Rotation. The orthogonal transformation matrix $\mathbf{A}_{j}$ from the body $j$ reference frame to the global reference frame can be written in terms of the transformation matrix $\mathbf{A}_{i j}^{\prime \prime}$ from the body $j$ reference frame $\left(x_{j}^{\prime} y_{j}^{\prime} z_{j}^{\prime}\right)$ to the joint reference frame of body $i\left(x_{i j}^{\prime \prime} y_{i j}^{\prime \prime} z_{i j}^{\prime \prime}\right)$, as $\mathbf{A}_{j}=\mathbf{A}_{i} \mathbf{C}_{i j} \mathbf{A}_{i j}^{\prime \prime}$. The variation of $\mathbf{A}_{j}$ is written as

$$
\delta \mathbf{A}_{j}=\delta \mathbf{A}_{i} \mathbf{C}_{i j} \mathbf{A}_{i j}^{\prime \prime}+\mathbf{A}_{i} \delta \mathbf{C}_{i j} \mathbf{A}_{i j}^{\prime \prime}+\mathbf{A}_{i} \mathbf{C}_{i j} \boldsymbol{\delta} \mathbf{A}_{i j}^{\prime \prime}
$$

Substituting for $\delta \mathbf{A}_{i}, \delta \mathbf{C}_{i j}$ into Eq. (22) and multiplying by the virtual rotation matrix yields

$$
\delta \tilde{\pi}_{j} \mathbf{A}_{j}=\delta \tilde{\pi}_{i} \mathbf{A}_{i} \mathbf{C}_{i j} \mathbf{A}_{i j}^{\prime \prime}+\mathbf{A}_{i} \delta \tilde{\xi}_{i j}^{\prime} \mathbf{C}_{i j} \mathbf{A}_{i j}^{\prime \prime}+\mathbf{A}_{i} \mathbf{C}_{i j} \delta \tilde{\pi}_{i j}^{\prime} \mathbf{A}_{i j}^{\prime \prime}
$$

Using the definition for $\delta \tilde{\pi}, \delta \mathbf{A}_{i j}^{\prime \prime}$ can be written as $\delta \mathbf{A}_{i j}^{\prime \prime}=\delta \tilde{\pi}_{i j}^{\prime} \mathbf{A}_{i j}^{\prime \prime}$. Substituting for $\delta \mathbf{A}_{i j}^{\prime \prime}$ yields $\mathbf{A}_{i} \mathbf{C}_{i j} \delta \tilde{\pi}_{i j}^{\prime}=\delta \tilde{\pi}_{i j} \mathbf{A}_{i} \mathbf{C}_{i j}$ where $\delta \pi_{i j}$ can be obtained from the relative coordinate variation $\delta \mathbf{q}_{j}$, such that

$$
\delta \pi_{i j}=\mathbf{H}_{j}\left(\mathbf{A}_{i}, \mathbf{q}_{j}\right) \delta \mathbf{q}_{j}
$$

and $\mathbf{H}_{j}\left(\mathbf{A}_{i}, \mathbf{q}_{j}\right)$ is a transformation matrix that depends on the orientation of body $i$ and on the relative coordinates $\delta \mathbf{q}_{j}$, which is defined for each type of joint. Using the definition of $\mathbf{A}_{j}$, Eq. (23) can be written as

$$
\delta \tilde{\pi}_{j} \mathbf{A}_{j}=\delta \tilde{\pi}_{i} \mathbf{A}_{j}+\delta \tilde{\xi}_{i j} \mathbf{A}_{j}+\delta \tilde{\pi}_{i j} \mathbf{A}_{j}
$$

Thus, the virtual rotation of body $j$ can be expressed as

$$
\delta \pi_{j}=\delta \pi_{i}+\delta \xi_{i j}+\delta \pi_{i j}
$$

Furthermore, substituting Eq. (24) into Eq. (26) yields the virtual rotation

$$
\delta \pi_{j}=\delta \pi_{i}+\delta \xi_{i j}+\mathbf{H}_{j} \delta \mathbf{q}_{j}
$$

3.2 State Vector Representation. Referring to Fig. 1, the origin of body $j$ reference frame can be located by the position vector given by 


$$
\mathbf{r}_{j}=\mathbf{r}_{i}+\mathbf{s}_{i j}+\mathbf{d}_{i j}
$$

Taking the variation of Eq. (28) yields

$$
\delta \mathbf{r}_{j}=\delta \mathbf{r}_{i}+\delta \mathbf{s}_{i j}+\delta \mathbf{d}_{i j}
$$

The vector $\mathbf{d}_{i j}$ can be written as $\mathbf{d}_{i j}=\mathbf{A}_{i} \mathbf{C}_{i j} \mathbf{d}_{i j}^{\prime \prime}\left(\mathbf{q}_{j}\right)$. Taking the variation of $\mathbf{d}_{i j}$ and substituting for $\delta \mathbf{A}_{i}$ and $\delta \mathbf{C}_{i j}$ yields

$$
\delta \mathbf{d}_{i j}=\delta \tilde{\pi}_{i} \mathbf{d}_{i j}-\tilde{\mathbf{d}}_{i j} \delta \xi_{i j}^{\prime}+\frac{\delta \mathbf{d}_{i j}}{\delta \mathbf{q}_{j}} \delta \mathbf{q}_{j}
$$

Substituting for $\delta \mathbf{d}_{i j}$ and $\delta \mathbf{s}_{i j}$ into Eq. (29) and using the relationship $\tilde{\mathbf{a}} \mathbf{b}=-\tilde{\mathbf{b}} \mathbf{a}$ yields

$$
\delta \mathbf{r}_{j}=\delta \mathbf{r}_{i}+\delta \tilde{\pi}_{i} \mathbf{s}_{i j}+\mathbf{A}_{i} \delta \mathbf{s}_{i j}^{\prime}+\delta \tilde{\pi}_{i} \mathbf{d}_{i j}-\widetilde{\mathbf{d}}_{i j} \delta \xi_{i j}^{\prime}+\frac{\delta \mathbf{d}_{i j}}{\delta \mathbf{q}_{j}} \delta \mathbf{q}_{j}
$$

Collecting terms and simplifying yields

$$
\delta \mathbf{r}_{j}=\delta \mathbf{r}_{i}+\delta \widetilde{\pi}_{i}\left(\mathbf{r}_{j}-\mathbf{r}_{i}\right)+\mathbf{A}_{i} \delta \mathbf{s}_{i j}^{\prime}-\widetilde{\mathbf{d}}_{i j} \delta \xi_{i j}^{\prime}+\frac{\delta \mathbf{d}_{i j}}{\delta \mathbf{q}_{j}} \delta \mathbf{q}_{j}
$$

Adding a common term $\left(\tilde{\mathbf{r}}_{j} \delta \pi_{j}\right)$ to both sides of Eq. (32), and using Eq. (27), Eq. (24), and the relationship $\tilde{\mathbf{a}} \mathbf{b}=-\tilde{\mathbf{b}} \mathbf{a}$ yields

$$
\delta \mathbf{r}_{j}+\widetilde{\mathbf{r}}_{j} \delta \pi_{j}=\delta \mathbf{r}_{i}+\widetilde{\mathbf{r}}_{i} \delta \pi_{i}+\mathbf{A}_{i} \delta \mathbf{s}_{i j}^{\prime}-\widetilde{\mathbf{d}}_{i j} \delta \xi_{i j}^{\prime}+\left(\frac{\delta \mathbf{d}_{i j}}{\delta \mathbf{q}_{j}}+\widetilde{\mathbf{r}}_{j} \mathbf{H}_{j}\right) \delta \mathbf{q}_{j}+\widetilde{\mathbf{r}}_{j} \delta \xi_{i j}^{\prime}
$$

Equation (48) and the virtual rotation of Eq. (41) can be combined in matrix form as

$$
\left[\begin{array}{c}
\delta \mathbf{r}_{j}+\tilde{\mathbf{r}}_{j} \delta \pi_{j} \\
\delta \pi_{j}
\end{array}\right]=\left[\begin{array}{c}
\delta \mathbf{r}_{i}+\tilde{\mathbf{r}}_{i} \delta \pi_{i} \\
\delta \pi_{i}
\end{array}\right]+\left[\begin{array}{c}
\mathbf{A}_{i} \\
\mathbf{0}
\end{array}\right] \delta \mathbf{s}_{i j}^{\prime}+\left[\begin{array}{c}
\frac{\partial \mathbf{d}_{i j}}{\partial \mathbf{q}_{j}}+\tilde{\mathbf{r}}_{j} \mathbf{H}_{j} \\
\mathbf{H}_{j}
\end{array}\right] \delta \mathbf{q}_{j}+\left[\begin{array}{c}
\tilde{\mathbf{r}}_{j}-\tilde{\mathbf{d}}_{i j} \\
\mathbf{I}
\end{array}\right] \delta \boldsymbol{\xi}_{i j}
$$

Equation (34) written in the state-vector notation is

$$
\delta \tilde{\mathbf{z}}_{j}=\delta \widehat{\mathbf{z}}_{i}+\mathbf{B}_{j} \delta \mathbf{q}_{j}+\mathbf{M}_{i} \delta \mathbf{s}_{i j}^{\prime}+\mathbf{N}_{j} \delta \xi_{i j}^{\prime}
$$

where the state variations of body $i$ and $j$ are defined as $\delta \hat{\mathbf{z}}_{i}=\left[\begin{array}{lll}\delta \mathbf{r}_{i}+\widetilde{\mathbf{r}}_{i} \delta \pi_{i} & \delta \pi_{i}\end{array}\right]^{T}$

and $\delta \tilde{\mathbf{z}}_{i}=\left[\begin{array}{ll}\delta \mathbf{r}_{j}+\widetilde{\mathbf{r}}_{j} \delta \pi_{j} & \delta \pi_{j}\end{array}\right]^{T}$, where $\mathbf{B}_{j}$ is the velocity transformation matrix written as $\mathbf{B}_{j}=\left[\begin{array}{ll}\frac{\partial \mathbf{d}_{i j}}{\partial \mathbf{q}_{j}}+\widetilde{\mathbf{r}}_{j} \mathbf{H}_{j} & \mathbf{H}_{j}\end{array}\right]^{T}$ and the virtual joint location transformation matrix is $\mathbf{M}_{i}=\left[\begin{array}{c}\mathbf{A}_{i} \\ \mathbf{0}\end{array}\right]$ and the virtual joint orientation transformation matrix between bodies $j$ and $i$ is $\mathbf{N}_{j}=\left[\begin{array}{ll}\widetilde{\mathbf{r}}_{j}-\tilde{\mathbf{d}}_{i j} & \mathbf{I}\end{array}\right]^{T}$. In order to represent the constraint variation in terms of state vector notation, define $\delta \mathbf{z}_{i}=\mathbf{T}_{i} \delta \hat{\mathbf{z}}_{i}$ and $\delta \mathbf{z}_{j}=\mathbf{T}_{j} \delta \hat{\mathbf{z}}_{j}$ where the virtual displacements are $\delta \mathbf{z}_{i}=\left[\begin{array}{ll}\delta \mathbf{r}_{i} & \delta \boldsymbol{\pi}_{i}\end{array}\right]^{T}$ and $\delta \mathbf{z}_{j}=\left[\begin{array}{ll}\delta \mathbf{r}_{j} & \delta \boldsymbol{\pi}_{j}\end{array}\right]^{T}$ and the transformation matrices $\mathbf{T}_{i}$ and $\mathbf{T}_{j}$ are defined as $\mathbf{T}_{i}=\left[\begin{array}{cc}\mathbf{I} & -\tilde{\mathbf{r}}_{i} \\ \mathbf{0} & \mathbf{I}\end{array}\right] \quad \mathbf{T}_{j}=\left[\begin{array}{cc}\mathbf{I} & -\tilde{\mathbf{r}}_{j} \\ \mathbf{0} & \mathbf{I}\end{array}\right]$, where $\mathbf{I}$ is the identity matrix. Substituting for $\delta \mathbf{z}_{i}$ and $\delta \mathbf{z}_{j}$ into Eq. (21) yields

$$
\delta \Phi=\Phi_{\mathbf{Z}_{i}} \mathbf{T}_{i} \delta \hat{\mathbf{z}}_{i}+\Phi_{\mathbf{Z}_{j}} \mathbf{T}_{j} \delta \hat{\mathbf{z}}_{j}+\Phi \xi^{i j} \delta \xi^{i j}+\Phi_{\mathbf{s}^{i j}} \delta \mathbf{s}^{i j}=\mathbf{0}
$$

The Jacobian of the constraint function can be written in terms of the state vector notation as 


$$
\Phi_{\hat{\mathbf{z}}_{i}}=\Phi_{\mathbf{z}_{i}} \mathbf{T}_{i} \text { and } \Phi_{\hat{\mathbf{z}}_{j}}=\Phi_{\mathbf{z}_{j}} \mathbf{T}_{j}
$$

Substituting Eqs. (37) into Eq. (36) yields the general expression for a constraint variation in a state vector representation as

$$
\delta \Phi=\Phi_{\hat{\mathbf{Z}}_{i}} \delta \hat{\mathbf{z}}_{i}+\Phi_{\hat{\mathbf{Z}}_{j}} \delta \hat{\mathbf{z}}_{j}+\Phi \xi^{i j} \delta \xi^{i j}+\Phi_{\mathbf{s}^{i j}} \delta \mathbf{s}^{i j}=\mathbf{0}
$$

In order to simplify the computation of the orientation, the virtual rotation $\delta \xi_{i j}$ is transformed to the virtual Euler parameters $\delta \mathbf{p}$ using the following transformation

$$
\delta \xi_{i j}=2 \mathbf{A}_{i} \mathbf{E}_{j} \delta \mathbf{p}_{j}
$$

where $\mathbf{p}_{j}=\left[\mathrm{e}_{j 0}, \mathrm{e}_{j l}, \mathrm{e}_{j 2}, \mathrm{e}_{j 3}\right]$ and $\mathbf{E}_{j}$ is the Euler parameter semi-rotation matrix defined as

$$
\mathbf{E}_{j}=\left[-\mathbf{e}_{j}, \tilde{\mathbf{e}}_{j}+\mathrm{e}_{j 0} \mathbf{I}\right]=\left[\begin{array}{cccc}
-\mathrm{e}_{1} & \mathrm{e}_{0} & -\mathrm{e}_{3} & \mathrm{e}_{2} \\
-\mathrm{e}_{2} & \mathrm{e}_{3} & \mathrm{e}_{0} & -\mathrm{e}_{l} \\
-\mathrm{e}_{3} & -\mathrm{e}_{2} & \mathrm{e}_{1} & \mathrm{e}_{0}
\end{array}\right]
$$

A normalization constraint given by

$$
\Phi_{j}=\mathbf{p}_{j}{ }^{\mathrm{T}} \mathbf{p}_{j}-1=\mathrm{e}_{0}{ }^{2}+\mathrm{e}_{1}{ }^{2}+\mathrm{e}_{2}{ }^{2}+\mathrm{e}_{3}{ }^{2}-1=0
$$

is imposed to restrict the four Euler parameters and to express the three relative degrees of freedom. In addition, the transformation matrix $\mathbf{C}_{i j}$ can be represented as $\mathbf{C}_{i j}=\mathbf{E}_{j} \mathbf{G}_{j}^{T}$ where $\mathbf{G}_{j}$ is the Euler parameter semi-rotation matrix defined by

$$
\mathbf{G}_{j}=\left[-\mathbf{e}_{j},-\tilde{\mathbf{e}}_{j}+\mathrm{e}_{j 0} \mathbf{I}\right]=\left[\begin{array}{cccc}
-\mathrm{e}_{1} & \mathrm{e}_{0} & \mathrm{e}_{3} & -\mathrm{e}_{2} \\
-\mathrm{e}_{2} & -\mathrm{e}_{3} & \mathrm{e}_{0} & \mathrm{e}_{1} \\
-\mathrm{e}_{3} & \mathrm{e}_{2} & -\mathrm{e}_{1} & \mathrm{e}_{0}
\end{array}\right]
$$

\section{Evaluating the Jacobian for a Cut constraint}

After cutting joints, the pair of bodies may become disconnected or remain connected by one joint. Therefore, two cases must be considered in evaluating the Jacobian matrix for a cut constraint.

4.1 Case 1: A Pair of Bodies Remains Connected by One Joint, after Cutting Additional Joints Between the Pair. Assume bodies $i$ and $j$ are connected by two kinematic joints. Choosing joint 1 to be cut, body $i$ is the inboard body of body $j$ (Fig. 2a).
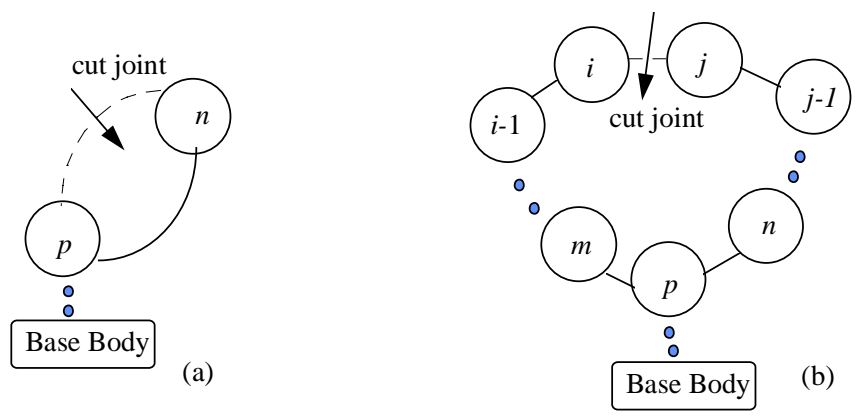

Fig. 2 After cutting a joint (a) a pair remains connected, (b) a pair is disconnected

In this case, the state variation of body $j$ can be represented in terms of the state variation of body $i$ by Eq. (35). Substituting Eq. (35) into Eq. (38) and recognizing that $\Phi_{\hat{\mathbf{Z}}_{i}}+\Phi_{\hat{\mathbf{Z}}_{j}}=\mathbf{0}$ yields

$$
\delta \Phi=\Phi_{\hat{\mathbf{z}}_{j}} \mathbf{B}_{j} \delta \mathbf{q}_{j}+\Phi_{\hat{\mathbf{z}}_{j}} \mathbf{M}_{i} \delta \mathbf{s}_{i j}^{\prime}+\Phi_{\hat{\mathbf{z}}_{j}} \mathbf{N}_{j} \delta \xi_{i j}^{\prime}+\Phi_{\mathbf{s}^{j}} \delta \mathbf{s}^{i j}+\Phi_{\xi^{j}} \delta \xi^{i j}
$$


4.2 A Pair of Bodies is Disconnected after Cutting Joints. Assume that the joint connecting bodies $i$ and $j$ is cut and that body $p$ is the junction node of the two chains that contain bodies $i$ and $j$, respectively (Fig. $2 \mathrm{~b}$ ). In this case, the state variation of body $j$ cannot be represented in terms of the state variation of body $i$ since no relative coordinates exist between bodies $i$ and $j$. Therefore, the state variations of bodies $i$ and $j$ must be written in terms of the state variation of their common junction node $p$ such that

$$
\delta \hat{\mathbf{z}}_{i}=\delta \hat{\mathbf{z}}_{i-1}+\mathbf{B}_{i} \delta \mathbf{q}_{i}+\mathbf{M}_{i-1} \delta \mathbf{s}_{i-1, i}^{\prime}+\mathbf{N}_{i} \delta \xi_{i-1, i}^{\prime}
$$

Equation (44) can be recursively used to obtain the state variation of body $i$ in terms of the state variation of its junction $p$ and those of the of relative coordinates along the chain as $\delta \tilde{\mathbf{z}}_{i}=\delta \tilde{\mathbf{z}}_{i-2}+\mathbf{B}_{i-1} \delta \mathbf{q}_{i-1}+\mathbf{M}_{i-2} \delta \mathbf{s}_{i-2, i-1}^{\prime}+\mathbf{N}_{i-1} \delta \xi_{i-2, i-1}^{\prime}+\mathbf{B}_{i} \delta \mathbf{q}_{i}+\mathbf{M}_{i-1} \delta \mathbf{s}_{i-2, i-1}^{\prime}+\mathbf{N}_{i} \delta \xi_{i-1, i}^{\prime}$

Simplifying and writing in terms of a summation yields

$$
\delta \tilde{\mathbf{z}}_{i}=\delta \hat{\mathbf{z}}_{p}+\sum_{k=m}^{i}\left(\mathbf{B}_{k} \delta \mathbf{q}_{k}+\mathbf{M}_{k-1} \delta \mathbf{s}_{k-1, k}^{\prime}+\mathbf{N}_{k} \delta \xi_{k-1, k}^{\prime}\right)
$$

Substituting for $\delta \tilde{\mathbf{z}}_{i}$ and $\delta \tilde{\mathbf{z}}_{j}$ into Eq. (61) yields

$$
\begin{aligned}
\delta \Phi & =\Phi_{\hat{\mathbf{z}}_{i}}\left[\delta \hat{\mathbf{z}}_{p}+\sum_{k=m}^{i}\left(\mathbf{B}_{k} \delta \mathbf{q}_{k}+\mathbf{M}_{k-1} \delta \mathbf{s}_{k-1, k}^{\prime}+\mathbf{N}_{k} \delta \xi_{k-1, k}^{\prime}\right)\right] \\
& +\Phi_{\hat{\mathbf{z}}_{j}}\left[\delta \hat{\mathbf{z}}_{p}+\sum_{k=n}^{j}\left(\mathbf{B}_{k} \delta \mathbf{q}_{k}+\mathbf{M}_{k-1} \delta \mathbf{s}_{k-1, k}^{\prime}+\mathbf{N}_{k} \delta \xi_{k-1, k}^{\prime}\right)\right]+\Phi_{\mathbf{s}^{i j}} \delta \mathbf{s}^{i j}+\Phi_{\xi^{i j}} \delta \xi^{i j}
\end{aligned}
$$

Collecting similar terms and rearranging yields

$$
\begin{aligned}
\delta \Phi= & \left(\Phi_{\hat{\mathbf{z}}_{i}}+\Phi_{\hat{\mathbf{z}}_{j}}\right) \delta \hat{\mathbf{z}}_{p}+\Phi_{\hat{\mathbf{z}}_{i}} \sum_{k=m}^{i}\left(\mathbf{B}_{k} \delta \mathbf{q}_{k}+\mathbf{M}_{k-1} \delta \mathbf{s}_{k-1, k}^{\prime}+\mathbf{N}_{k} \delta \xi_{k-1, k}^{\prime}\right) \\
& +\Phi_{\hat{\mathbf{z}}_{j}} \sum_{k=n}^{j}\left(\mathbf{B}_{k} \delta \mathbf{q}_{k}+\mathbf{M}_{k-1} \delta \mathbf{s}_{k-1, k}^{\prime}+\mathbf{N}_{k} \delta \xi_{k-1, k}^{\prime}\right)+\Phi_{\mathrm{s}^{j}} \delta \mathbf{s}^{i j}+\Phi_{\xi^{i j}} \delta \xi^{i j}
\end{aligned}
$$

where $\Phi_{\hat{\mathbf{z}}_{i}}+\Phi_{\hat{\mathbf{z}}_{i}}=\mathbf{0}$. The constraint variation, written in terms of the Jacobian is

$$
\delta \Phi=\Phi_{\mathbf{q}} \delta \mathbf{q}
$$

where $\Phi_{\mathbf{q}}=\left[\Phi_{\hat{\mathbf{Z}}_{i}} \mathbf{B}_{m} \ldots \Phi_{\hat{\mathbf{Z}}_{i}} \mathbf{B}_{i}\left|\Phi_{\hat{\mathbf{Z}}_{i}} \mathbf{M}_{m-1} \ldots \Phi_{\hat{\mathbf{Z}}_{i}} \mathbf{M}_{i-1}\right| \Phi_{\hat{\mathbf{Z}}_{i}} \mathbf{N}_{m} \ldots \Phi_{\hat{\mathbf{Z}}_{i}} \mathbf{N}_{i} \mid \Phi_{\hat{\mathbf{Z}}_{j}} \mathbf{B}_{n} \ldots\right.$

$$
\left.\Phi_{\hat{\mathbf{Z}}_{j}} \mathbf{B}_{j}\left|\Phi_{\hat{\mathbf{Z}}_{j}} \mathbf{M}_{n-1} \ldots \Phi_{\hat{\mathbf{Z}}_{j}} \mathbf{M}_{j-l}\right| \Phi_{\hat{\mathbf{Z}}_{j}} \mathbf{N}_{n} \ldots \Phi_{\hat{\mathbf{Z}}_{j}} \mathbf{N}_{j}\left|\Phi_{\mathbf{S}^{i}}\right| \Phi \xi^{i j}\right]
$$

and

$$
\begin{array}{r}
\delta \mathbf{q}=\left[\delta \mathbf{q}_{m} \ldots \delta \mathbf{q}_{i}\left|\delta \mathbf{s}_{m-1, m}^{\prime} \ldots \delta \mathbf{s}_{i-1, i}^{\prime}\right| \delta \xi_{m-1, m}^{\prime} \ldots \delta \xi_{i-1, i}^{\prime}\left|\delta \mathbf{q}_{n} \ldots \delta \mathbf{q}_{j}\right|\right. \\
\left.\delta \mathbf{s}_{n-1, n}^{\prime} \ldots \delta \mathbf{s}_{j-1, j}^{\prime}\left|\delta \xi_{n-1, n}^{\prime} \ldots \delta \xi_{j-1, j}^{\prime}\right| \delta \mathbf{s}^{i j} \mid \delta \xi^{i j}\right]^{T}
\end{array}
$$

The numerical algorithm used to compute an assembled configuration due to a design propagation is depicted in Fig. 3. 
Fig. 3 Algorithm for computing kinematic design propagations

In order to validate the foregoing analysis and to demonstrate the numerical solutions, a spatial mechanism is analyzed using the presented formulation. Section 5 will illustrate the formulation using the analysis of a the McPherson strut suspension system.

\section{Numerical Example}

The McPherson strut suspension, shown in Fig. 4a, has been simplified as a two-body system comprising a chassis and a wheel assembly with three joints. Since the mass of the lower control arm, strut, rack and tie rod are insignificant compared with the chassis or the wheel assembly, they are usually modeled as composite joints. The model comprises a composite RevoluteSpherical (R-S) joint, a composite Spherical-Translational (S-T) joint, and a composite Translational-Distance (T-D) joint connecting the chassis and the wheel assembly.

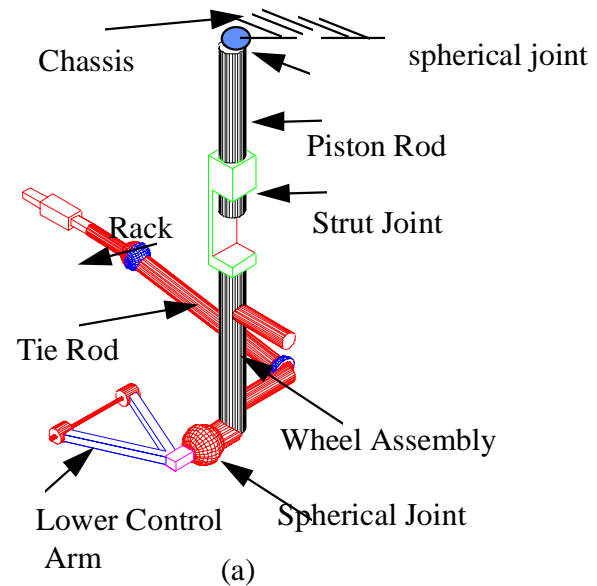

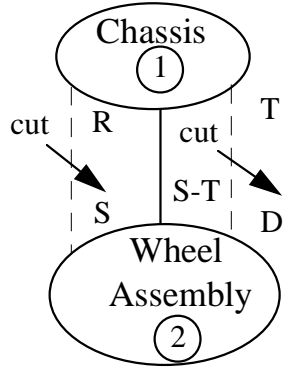

(b)

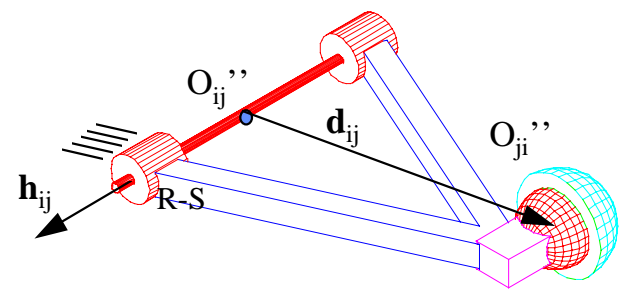

(c)

Fig. 4 (a) McPherson strut suspension system (definition of reference frames) (b) Graph representation of the system and (c) Revolute-sppherical joint

The McPherson strut suspension system has two degrees of freedom with respect to the chassis, i.e., jounce and steering. To form a spanning tree structure, the composite R-S and the composite T-D joints are cut. The system belongs to case 1 and the graph representation is shown in Fig. 4b.

The R-S joint, shown in Fig. 4c, connects the chassis by a revolute joint and the wheel assembly by a spherical joint. It has four relative degrees-of-freedom, one for the revolute and three for the spherical joint. Thus, it has two constraint equations.

where partial derivatives of the variation of the basic constraints (Eq. 7, 14, 16, and 18), written in terms of their coefficients, are presented in Table 1. 
Table 1 Partial Derivatives of Basic Constraint Functions

\begin{tabular}{|c|c|c|c|c|c|c|c|c|}
\hline $\begin{array}{c}\text { Constraint } \\
\text { Function }\end{array}$ & $\Phi_{\mathbf{r}_{i}}$ & $\Phi_{\mathbf{r}_{j}}$ & $\Phi_{\pi_{i}}$ & $\Phi_{\pi_{j}}$ & $\Phi_{s_{i j}^{\prime}}$ & $\Phi_{\mathbf{s}_{j i}^{\prime}}$ & $\Phi_{\xi_{j i}^{\prime}}$ & $\Phi_{\xi_{j i}^{\prime}}$ \\
\hline $\begin{array}{c}\text { Dot-1 } \\
\Phi^{d 1}\left(\mathbf{a}_{i}, \mathbf{a}_{j}\right)\end{array}$ & $\mathbf{0}$ & $\mathbf{0}$ & $-\mathbf{a}_{j}{ }^{T} \tilde{\mathbf{a}}_{i}$ & $-\mathbf{a}_{i}{ }^{T} \tilde{\mathbf{a}}_{j}$ & $\mathbf{0}$ & $\mathbf{0}$ & $-\mathbf{a}_{j}{ }_{j}^{T} \tilde{\mathbf{a}}_{i}$ & $-\mathbf{a}_{i}{ }^{T} \tilde{\mathbf{a}}_{j}$ \\
\hline $\begin{array}{c}\text { Dot-2 } \\
\Phi^{d 2}\left(\mathbf{a}_{i}, \mathbf{d}_{i j}\right)\end{array}$ & $-\mathbf{a}_{i}{ }^{T}$ & $\mathbf{a}_{i}{ }^{T}$ & $\left(\mathbf{a}_{i}^{T} \widetilde{\mathbf{s}}_{i j}-\mathbf{d}_{i j}^{T} \widetilde{\mathbf{a}}_{i}\right)$ & $-\mathbf{a}_{i}{ }^{T} \tilde{\mathbf{s}}_{j i}$ & $-\mathbf{a}_{i}{ }^{T} \mathbf{A}_{i}$ & $\mathbf{a}_{i}{ }^{T} \mathbf{A}_{j}$ & $-\mathbf{d}_{i j}^{T} \tilde{\mathbf{a}}_{i}$ & $\mathbf{0}$ \\
\hline $\begin{array}{c}\text { Spherical } \\
\Phi^{s}\left(O_{i j}^{\prime \prime}, O_{j i}^{\prime \prime}\right)\end{array}$ & $-\mathbf{I}$ & $\mathbf{I}$ & $\tilde{\mathbf{s}}_{i j}$ & $-\tilde{\mathbf{s}}_{j i}$ & $-\mathbf{A}_{i}$ & $\mathbf{A}_{j}$ & $\mathbf{0}$ & $\mathbf{0}$ \\
\hline $\begin{array}{c}\text { Distance } \\
\Phi^{d i s}\left(O_{i j}^{\prime \prime}, O_{j i}^{\prime \prime}, \ell\right.\end{array}$ & $-2 \mathbf{d}_{i j}^{T}$ & $2 \mathbf{d}_{i j}^{T}$ & $2 \mathbf{d}_{i j}^{T} \tilde{\mathbf{s}}_{i j}$ & $-2 \mathbf{d}_{i j}^{T} \tilde{\mathbf{s}}_{j i}$ & $-2 \mathbf{d}_{i j}^{T} \mathbf{A}_{i}$ & $2 \mathbf{d}_{i j}^{T} \mathbf{A}_{j}$ & $\mathbf{0}$ & $\mathbf{0}$ \\
\hline
\end{tabular}

\section{SPATIAL SLIDER-CRANK MECHANISM}

Consider the spatial slider crank mechanism shown in Fig. 4, having a revolute joint, a universal joint, and a translational joint.
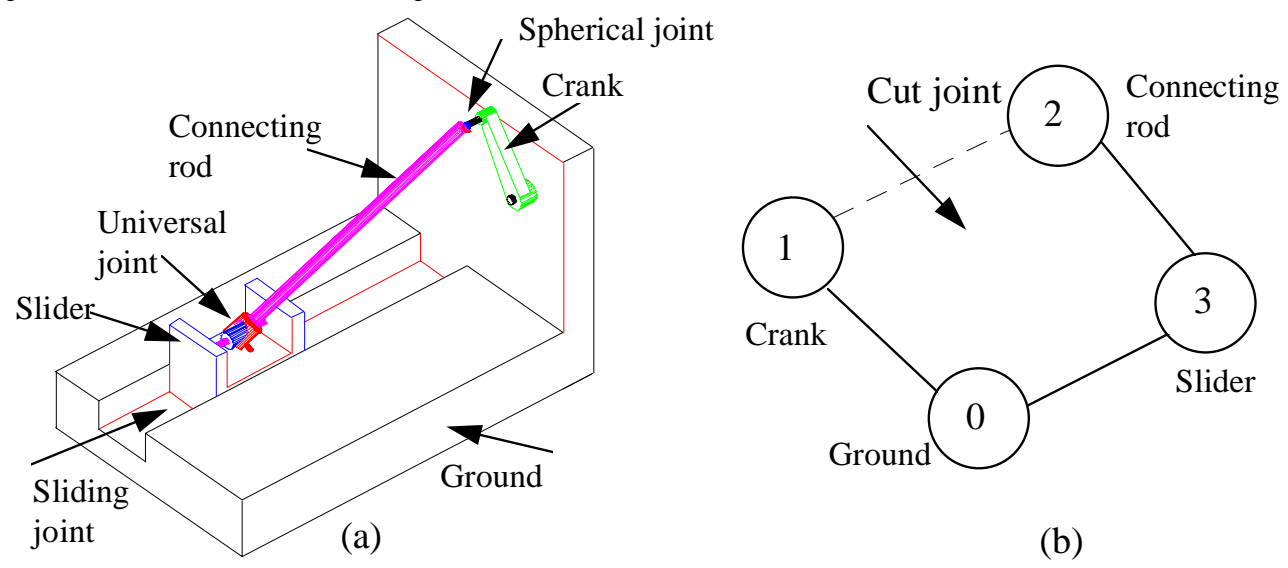

Fig. 4 Spatial slider-crank mechanism

This system has four relative generalized coordinates $\mathbf{q}=\left[\begin{array}{llll}q_{1} & q_{2} & q_{3} & q_{4}\end{array}\right]^{\mathrm{T}}, q_{1}$ for the revolute joint, $q_{2}$ and $q_{3}$ for the universal joint, and $q_{4}$ for the translation joint. To form a spanning tree, the spherical joint is cut, as illustrated in Fig. 4b. The system has three scalar constraint equations.

$$
\Phi^{s p h}=\mathbf{r}_{2}+\mathbf{S}_{21}-\mathbf{S}_{12}-\mathbf{r}_{1}=\mathbf{0}
$$

The tree graph represents a pair of links that are disconnected after cutting a joint (case 1). For a given initial estimate that does not satisfy the constraint function $\Phi$, the set of linear equations written as

$$
\Phi_{\mathbf{q}}\left(\mathbf{q}^{(k)}\right) \delta \mathbf{q}^{(k)}=-\Phi\left(\mathbf{q}^{(k)}\right)
$$


are solved simultaneously for a correction $\delta \mathbf{q}^{(\mathrm{k})}$. The corrected term is added to the estimate $\mathbf{q}^{(\mathrm{k})}$ to obtain an improved estimate such that

$$
\mathbf{q}^{(k+1)}=\mathbf{q}^{(k)}+\delta \mathbf{q}^{(k)}
$$

In this example, the linear equations are written as

$$
\Phi_{\mathbf{q}}^{s p h} \delta \mathbf{q}=\Phi^{s p h}
$$

where $\Phi_{\mathbf{q}}^{\text {sph }}$ is the Jacobian matrix in joint coordinate space. The Jacobian matrix $\Phi_{\hat{\mathbf{z}}}^{\text {sph }}$ in cartesian space can be transformed to joint space using velocity transformations as

$$
\Phi_{\mathbf{q}}^{\text {sph }}=\left[\Phi_{\hat{\mathbf{z}}}^{\text {sph }} \mathbf{B}_{\text {rev }}\left|\Phi_{\hat{\mathbf{z}}}^{\text {sph }} \mathbf{B}_{\text {trans }}\right| \Phi_{\hat{\mathbf{z}}}^{\text {sph }} \mathbf{B}_{\text {uni }}\right]
$$

where $\mathbf{B}_{\text {rev }}$ is the transformation matrix for the revolute joint

and $\mathbf{B}_{\text {uni }}$ is for the universal joint

$$
\begin{gathered}
\mathbf{B}_{\mathrm{rev}}=\left[\begin{array}{c}
\tilde{\mathbf{r}}_{I} \mathbf{h}_{o l} \\
\mathbf{h}_{0 l}
\end{array}\right] \\
\mathbf{h}_{01}=\mathbf{A}_{0} \mathbf{C}_{01}\left[\begin{array}{lll}
0 & 0 & 1
\end{array}\right]^{T}
\end{gathered}
$$

$$
\begin{gathered}
\mathbf{B}_{\text {uni }}=\left[\begin{array}{cc}
\tilde{\mathbf{r}}_{2} \mathbf{h}_{32} & \tilde{\mathbf{r}}_{2} \mathbf{g}_{32} \\
\mathbf{h}_{32} & \mathbf{g}_{32}
\end{array}\right] \\
\mathbf{h}_{32}=\mathbf{A}_{3} \mathbf{C}_{32}\left[\begin{array}{lll}
0 & 0 & 1
\end{array}\right]^{T} \\
\mathbf{g}_{32}=\mathbf{A}_{3} \mathbf{C}_{32} \mathbf{A}_{321}^{\prime \prime}\left(q_{2}\right)\left[\begin{array}{lll}
0 & 0 & 1
\end{array}\right]^{T}
\end{gathered}
$$

where the rotation matrix is

$$
\mathbf{A}_{321} "\left(\mathrm{q}_{2}\right)=\left[\begin{array}{ccc}
\cos \left(\mathrm{q}_{2}\right) & -\sin \left(\mathrm{q}_{2}\right) & 0 \\
\sin \left(\mathrm{q}_{2}\right) & \cos \left(\mathrm{q}_{2}\right) & 0 \\
0 & 0 & 1
\end{array}\right]
$$

For the translational joint, the velocity transformation matrix is $\mathbf{B}_{\text {trans }}$ defined as

$$
\begin{gathered}
\mathbf{B}_{\text {trans }}=\left[\begin{array}{c}
\mathbf{g}_{03} \\
\mathbf{0}
\end{array}\right] \\
\mathbf{g}_{01}=\mathbf{A}_{0} \mathbf{C}_{03}\left[\begin{array}{lll}
0 & 0 & 1
\end{array}\right]^{T}
\end{gathered}
$$

where

$$
\begin{gathered}
\Phi_{\widehat{\mathbf{z}}_{i}}^{\mathrm{sph}}=\left[\begin{array}{ll}
-\mathbf{I} & \widetilde{\mathbf{r}}_{1}+\widetilde{\mathbf{s}}_{12}
\end{array}\right] \\
\Phi_{\hat{\mathbf{z}}_{j}}^{\mathrm{sph}}=\left[\begin{array}{ll}
-\mathbf{I} & -\left(\widetilde{\mathbf{r}}_{2}+\widetilde{\mathbf{s}}_{21}\right)
\end{array}\right]
\end{gathered}
$$

The initial estimates of the position and orientations of the ground are presented in Table 2 .

TABLE 2

Position and Orientation Estimates of the Ground

\begin{tabular}{|c|cccccc|}
\hline body & $\mathrm{x}$ & $\mathrm{y}$ & $\mathrm{z}$ & $\mathrm{e}_{1}$ & $\mathrm{e}_{2}$ & $\mathrm{e}_{3}$ \\
ground & 0 & 0 & 0 & 0 & 0 & 0 \\
\hline
\end{tabular}

Initial estimates of joint attachment vectors in the body reference frame are presented in Table 3.

TABLE 3

Joint Attachment vectors in Body Reference Frame Estimates

\begin{tabular}{|llll|llll|}
\hline $\mathrm{s}^{\prime}$ & $\mathrm{x}^{\prime}$ & $\mathrm{y}^{\prime}$ & $\mathrm{z}^{\prime}$ & $\mathrm{y}^{\prime}$ & $\mathrm{x}^{\prime}$ & $\mathrm{y}^{\prime}$ & $\mathrm{z}$ \\
\hline
\end{tabular}




\begin{tabular}{|llll|lllc|}
\hline $\mathbf{s}_{o 1}^{\prime}$ & 0 & 0 & 0 & $\mathbf{S}_{o 3}^{\prime}$ & 0 & 3.8637 & 0 \\
$\mathbf{S}_{12}^{\prime}$ & 2 & 0 & 0 & $\mathbf{S}_{32}$ & 0 & 0 & 0 \\
$\mathbf{s}_{21}^{\prime}$ & -2.828 & 0 & 0 & & & & \\
\hline
\end{tabular}

Transformation Matrices from Joint Coordinates to the Body Reference Frame are as follows.
$\mathbf{C}_{01}=\left[\begin{array}{lll}0 & 0 & 1 \\ 1 & 0 & 0 \\ 0 & 1 & 0\end{array}\right]$
$\mathbf{C}_{03}=\left[\begin{array}{lll}0 & 1 & 0 \\ 0 & 0 & 1 \\ 1 & 0 & 0\end{array}\right]$
$\mathbf{C}_{32}=\left[\begin{array}{lll}0 & 1 & 0 \\ 0 & 0 & 1 \\ 1 & 0 & 0\end{array}\right]$

Estimates of generalized coordinates are $\mathbf{q}=\left[\begin{array}{llll}45^{\circ} & -30^{\circ} & 0^{\circ} & 0^{\circ}\end{array}\right]^{T}$.

For a given initial estimate, the Newton-Raphson iteration method is used until the constraint violations are satisfied. A linkage length or a transformation matrix from joint coordinate to body reference frame may be changed. Therefore, joint attachment vectors may be changed (e.g., $\mathbf{s}_{o l}$, the revolute joint attachment vector in ground reference frame.) Once the linkage length has been changed, the constraint function is violated, and three options can be performed: (1) The generalized coordinates $\mathbf{q}$ are changed such that

$$
\Phi_{\hat{\mathbf{z}}_{l}}{ }^{\text {sph }} \mathbf{B}_{\text {rev }} \delta \mathrm{q}_{1}+\Phi_{\hat{\mathbf{z}}_{2}}{ }^{\text {sph }} \mathbf{B}_{\text {trans }} \delta \mathrm{q}_{4}+\Phi_{\hat{\mathbf{z}}_{2}}{ }^{\text {sph }} \mathbf{B}_{\text {uni }}\left[\begin{array}{l}
\delta \mathrm{q}_{2} \\
\delta \mathrm{q}_{3}
\end{array}\right]=-\Phi
$$

(2) The linkage lengths $\mathbf{s}^{\prime}$ are changed such that

$\Phi_{\mathbf{z}_{1}}{ }^{\mathrm{sph}} \mathbf{M}_{0} \delta \mathbf{s}_{01}^{\prime}+\Phi_{\hat{\mathbf{z}}_{2}}{ }^{\mathrm{sph}} \mathbf{M}_{0} \delta \mathbf{s}_{03}^{\prime}+\Phi_{\mathbf{z}_{2}}{ }^{\mathrm{sph}} \mathbf{M}_{3} \delta \mathbf{s}_{32}^{\prime}+\Phi_{\mathbf{s}_{12}} \delta \mathbf{s}_{12}^{\prime}+\Phi_{\mathbf{s}_{2 l}} \delta \mathbf{s}_{21}^{\prime}=-\Phi$

where

$$
\mathbf{M}_{0}=\left[\begin{array}{c}
\mathbf{A}_{0} \\
\mathbf{0}
\end{array}\right] \quad \mathbf{M}_{3}=\left[\begin{array}{c}
\mathbf{A}_{3} \\
\mathbf{0}
\end{array}\right]
$$

$\Phi_{s_{12}}$ and $\Phi_{s_{2 l}}$ are spherical constraint given in Table 1 .

(3) The generalized coordinates $\mathbf{q}$ and linkage lengths $\mathbf{s}$ are changed such that

$$
\begin{aligned}
& \Phi_{\hat{\mathbf{z}}_{l}}{ }^{\text {sph }} \mathbf{B}_{\text {rev }} \delta \mathrm{q}_{l}+\Phi_{\hat{\mathbf{z}}_{l}}{ }^{\text {sph }} \mathbf{M}_{0} \delta \mathbf{s}_{0 l}{ }^{\prime}+\Phi_{\hat{\mathbf{z}}_{2}}{ }^{\mathrm{sph}} \mathbf{B}_{\mathrm{trans}} \delta \mathrm{q}_{4}+\Phi_{\hat{\mathbf{z}}_{2}}{ }^{\mathrm{sph}} \mathbf{B}_{\text {uni }}\left[\begin{array}{l}
\delta \mathrm{q}_{2} \\
\delta \mathrm{q}_{3}
\end{array}\right]+\Phi_{\hat{\mathbf{z}}_{2}}{ }^{\text {sph }} \mathbf{M}_{\delta} \delta \mathbf{s}_{03}{ }_{03} \\
& +\Phi_{\mathbf{z}_{2}}^{\text {sph }} \mathbf{M}_{3} \delta \mathbf{s}_{32}^{\prime}+\Phi_{\mathbf{s}_{12}} \delta \mathbf{s}_{12}^{\prime}+\Phi_{\mathbf{s}_{2 l}} \delta \mathbf{s}_{2 l}^{\prime}=-\Phi
\end{aligned}
$$

Initial estimates are provided for an assembled configuration. The simulation results due to changing the spherical joint attachment vector in crank reference frame $\mathbf{s}_{21}$ from $\left[\begin{array}{lll}-2.828 & 0 & 1\end{array}\right]^{T}$ to $\left[\begin{array}{lll}-2.8 & 0.2 & 0.1\end{array}\right]^{T}$ are presented in Table 4. Results of propagating the

\begin{tabular}{|c|c|c|c|}
\hline variables & $\mathbf{q}$ & $\mathbf{s}_{\mathrm{ij}}^{\prime}$ & $\mathbf{q}$ and $\mathbf{s}_{\mathrm{ij}}$ \\
\hline $\mathbf{S}_{o l}^{\prime}$ & {$\left[\begin{array}{lll}0 & 0 & 0\end{array}\right]^{\mathrm{T}}$} & $\begin{array}{c}{\left[\begin{array}{ll}0.025 & 0.0311538 \\
0.0397479\end{array}\right]^{\mathrm{T}}} \\
{ }^{2}\end{array}$ & 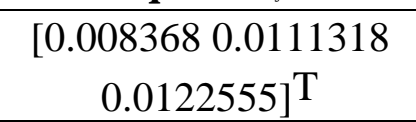 \\
\hline $\mathbf{S}_{03}^{\prime}$ & {$\left[\begin{array}{llll}0 & 3.8637 & 0\end{array}\right]^{\mathrm{T}}$} & $\begin{array}{c}{\left[\begin{array}{cc}-0.025 & 3.83255 \\
0.0397479\end{array}\right]^{\mathrm{T}}} \\
0\end{array}$ & $\begin{array}{c}{[-0.0083683 .85257-} \\
0.0111318]^{\mathrm{T}}\end{array}$ \\
\hline $\mathbf{s}_{32}$ & {$\left[\begin{array}{lll}0 & 0 & 0\end{array}\right]^{\mathrm{T}}$} & $\begin{array}{c}-0.0397479-0.025- \\
0.0311538]^{\mathrm{T}}\end{array}$ & $\begin{array}{c}-0.012255-0.008368- \\
0.0111318]^{\mathrm{T}}\end{array}$ \\
\hline
\end{tabular}
change in this design are illustrated in Fig. 5.

\section{TABLE 4}

Simulation results due to a change in $\mathbf{S}_{21}$ 


\begin{tabular}{|c|c|c|c|}
\hline $\mathbf{s}_{12}^{\prime}$ & {$\left[\begin{array}{lll}2 & 0 & 0\end{array}\right]^{\mathrm{T}}$} & $\begin{array}{c}{\left[\begin{array}{cc}2.05014 & 0.00607691 \\
0.025\end{array}\right]^{\mathrm{T}}}\end{array}$ & $\begin{array}{c}{[2.016540 .000794346} \\
0.008368]^{\mathrm{T}}\end{array}$ \\
\hline $\mathbf{S}_{21}^{\prime}$ & {$\left[\begin{array}{llll}-2.8 & 0.2 & 0.1\end{array}\right]^{\mathrm{T}}$} & {$\left[\begin{array}{llll}-2.8 & 0.2 & 0.1\end{array}\right]^{\mathrm{T}}$} & {$\left[\begin{array}{lll}-2.8 & 0.2 & 0.1\end{array}\right]^{\mathrm{T}}$} \\
\hline $\mathrm{q}_{l}$ & 44.893707 & 45 & 45.090633 \\
\hline $\mathrm{q}_{2}, \mathrm{q}_{3}$ & $\begin{array}{c}-26.0852,- \\
2.0454\end{array}$ & $-30,0$ & $-27.367386,-1.3609008$ \\
\hline $\mathrm{q}_{4}$ & -0.0184005 & 0 & -0.0111318 \\
\hline$\Phi$ & $1.26576 \mathrm{e}-05$ & $1.11076 \mathrm{e}-16$ & $\begin{array}{c}1.1 \\
321 \mathrm{e}-06 \\
\end{array}$ \\
\hline Iterations & 2 & 1 & 2 \\
\hline
\end{tabular}

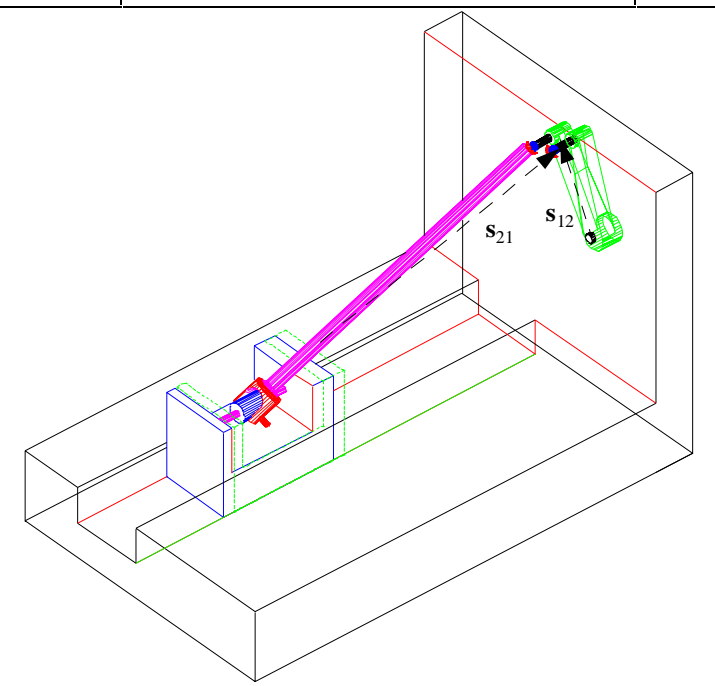

Fig. 5 Changes in the mechanism due to a design propagation

It is required that the vector $\mathbf{d}_{i j} \neq \mathbf{0}$ between joint definition points $O_{i j}^{\prime \prime}$ and $O_{j i}^{\prime \prime}$, be orthogonal to the axis of rotation of the revolute joint $\mathbf{h}_{i j}$ (a unit vector along the axis of rotation of the revolute joint). It is also required that the distance between the origins $O_{i j}^{\prime \prime}$ and $O_{j i}^{\prime \prime}$ (lower control arm length $\ell_{R-S}$ ) be equal to $\ell_{R-S} \neq 0$. The two additional constraints are $\Phi^{d 2}\left(\mathbf{h}_{i j}, \mathbf{d}_{i j}\right)=0$ and $\Phi^{d i s}\left(O_{i j}^{\prime \prime}, O_{i j}^{\prime \prime}, l_{R-S}\right)=0$. The two constraints are combined into one set as

$$
\Phi^{R-S}=\left[\begin{array}{c}
\Phi^{d 2} \\
\Phi^{d i s}
\end{array}\right]=\left[\begin{array}{c}
\mathbf{h}_{i j}^{T} \mathbf{d}_{i j} \\
\mathbf{d}_{i j}^{T} \mathbf{d}_{i j}-l_{R-S}^{2}
\end{array}\right]=\mathbf{0}
$$

where $\mathbf{h}_{i j}$ can be written as $\mathbf{h}_{i j}=\mathbf{h}_{12 R-S}=\mathbf{A}_{1} \mathbf{C}_{12 R-S}\left[\begin{array}{lll}0 & 0 & 1\end{array}\right]^{T}$ and the distance $\mathbf{d}_{i j}$ can be written as $\mathbf{d}_{i j}=\mathbf{d}_{12 R-S}=\mathbf{r}_{2}+\mathbf{s}_{21 R-S}-\mathbf{r}_{1}+\mathbf{s}_{12 R-S}$ where the subscript $21 R-S$ denotes the R-S joint between body chassis and wheel assembly. The Jacobian matrix of the constraint function of Eq. (52) in Cartesian space can be written as $\Phi_{\hat{\mathbf{z}}_{j}}^{R-S}=\left[\begin{array}{ll}\Phi_{\hat{\mathbf{z}}_{j}}^{d 2} & \Phi_{\hat{\mathbf{z}}_{j}}^{d i s}\end{array}\right]^{T}$, where $\Phi_{\tilde{\mathbf{z}}_{j}}^{d 2}=\left[\mathbf{h}_{i j}^{T}-\mathbf{h}_{i j}^{T}\left(\widetilde{\mathbf{r}}_{j}+\widetilde{\mathbf{s}}_{j i}\right)\right] \quad$ and
$\Phi_{\tilde{\mathbf{z}}_{j}}^{d i s}=2 \mathbf{d}_{i j}^{T}\left[\mathbf{I}-\left(\widetilde{\mathbf{r}}_{j}+\widetilde{\mathbf{s}}_{j i}\right)\right]$, where $\mathbf{r}_{j}=\mathbf{r}_{2}=\mathbf{r}_{1}+\mathbf{s}_{12 S-T}+\mathbf{d}_{12 S-T}$. 
and the vector $\mathbf{d}_{12 S-T}$ can be written as $\mathbf{d}_{12 S-T}=\mathbf{g}_{i j} q_{2} \quad$ and $\quad \mathbf{g}_{i j}=\mathbf{A}_{1} \mathbf{C}_{12 S-T} \mathbf{A}_{s p h}^{\prime \prime}\left[\begin{array}{lll}0 & 0 & 1\end{array}\right]^{T}$, where $\mathbf{A}_{\text {sph }}^{\prime \prime}$ is the transformation matrix of the spherical joint reference frame between wheel assembly and chassis. Since the T-D joint, connects the chassis by a translational joint and the wheel assembly by a distance joint, it introduces an additional relative degree-of-freedom which is the relative translational coordinate $\mathrm{q}_{3}$. A new vector $\mathbf{I}_{i j}$ is introduced (as shown in Fig. 5) such that $\mathbf{l}_{i j}=\mathbf{d}_{i j}-\mathbf{h}_{i j} q_{3}$. The constraint equation that characterizes this joint is

$$
\Phi^{T-D}=\mathbf{l}_{i j}^{T} \mathbf{I}_{i j}-l_{T-D}^{2}=0
$$

where $\mathbf{d}_{i j}=\mathbf{d}_{12 T-D}-\mathbf{r}_{l}-\mathbf{s}_{12 T-D}$ and $\mathbf{h}_{12 T-D}=\mathbf{A}_{l} \mathbf{C}_{12 T-D}\left[\begin{array}{lll}0 & 0 & 1\end{array}\right]^{T}$.

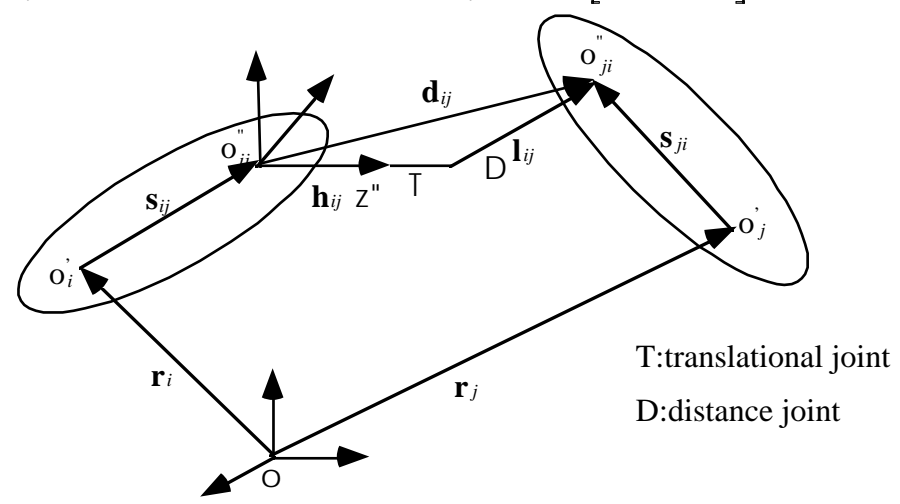

Fig. 5 Translational-distance joint

Taking the variation of Eq. (53) yields

$$
\begin{array}{r}
\delta \Phi^{T-D}=2 \mathbf{l}_{i j}^{T}\left[\delta \mathbf{r}_{j}+\mathbf{A}_{j} \delta \mathbf{s}_{j i}^{\prime}-\widetilde{\mathbf{s}}_{j i} \delta \pi_{j}-\delta \mathbf{r}_{i}-\mathbf{A}_{i} \delta \mathbf{s}_{i j}^{\prime}+\left(\widetilde{\mathbf{s}}_{i j}+\tilde{\mathbf{h}}_{i j} q_{3}\right) \delta \pi_{i}\right. \\
\left.+\tilde{\mathbf{h}}_{i j} q_{3} \delta \xi_{i j}-\mathbf{h}_{i j} \delta q_{3}\right]-2 l_{T-D} \delta l_{T-D}
\end{array}
$$

The Jacobian matrix of the T-D constraint in Cartesian space is

$$
\Phi_{\tilde{\mathbf{z}}_{j}}^{T-D}=\left[\begin{array}{ll}
\Phi_{\mathbf{r}_{j}} & \Phi_{\pi_{j}}
\end{array}\right]\left[\begin{array}{cc}
\mathbf{I} & -\widetilde{\mathbf{r}}_{j} \\
\mathbf{0} & \mathbf{I}
\end{array}\right]
$$

and can be written as

$$
\Phi_{\hat{\mathbf{z}}_{j}}^{T-D}=\left[\begin{array}{ll}
2 \mathbf{l}_{i j}^{T} & -2 \mathbf{l}_{i j}^{T}\left(\widetilde{\mathbf{s}}_{j i}+\widetilde{\mathbf{r}}_{j}\right)
\end{array}\right]
$$

For the T-D joint, the Jacobian matrix is

$$
\Phi_{q_{3}}^{T-D}=-2 \mathbf{l}_{i j}^{T} \mathbf{h}_{i j}
$$

The uncut S-T joint connects the chassis with a spherical joint and the wheel assembly with a translational joint. It has four relative degrees of freedom $\mathbf{q}_{S-T}=\left[\begin{array}{ll}\mathbf{q}_{1} & q_{2}\end{array}\right]^{T}$, three for the spherical joint and one for the translational joint. Three relative rotational coordinates of the spherical joint can be expressed using Euler parameters to avoid orientation-associated singularities. The Euler parameters are denoted by $\mathrm{e}_{0}, \mathrm{e}_{1}, \mathrm{e}_{2}, \mathrm{e}_{3}$ such that $\mathbf{p}_{1}=\left[\begin{array}{llll}\mathrm{e}_{0} & \mathrm{e}_{1} & \mathrm{e}_{2} & \mathrm{e}_{3}\end{array}\right]^{T}$ and the normalization $\Phi_{j}=\mathbf{p}_{1}^{T} \mathbf{p}_{1}-1=\mathrm{e}_{0}^{2}+\mathrm{e}_{1}^{2}+\mathrm{e}_{2}^{2}+\mathrm{e}_{3}^{2}-1=0$ is imposed (Eq. 41).

Since a translational joint does not have a rotational degree of freedom, the transformation matrix $\mathbf{A}_{i j}^{\prime \prime}$ of the S-T joint is simply the same as that of a spherical joint, thus $\quad \mathbf{A}_{i j}^{\prime \prime}=\mathbf{A}_{s p h}^{\prime \prime}=\mathbf{E}_{j} \mathbf{G}_{j}^{T}$. The velocity transformation matrix $\mathbf{B}_{j}$ can be expressed as 


$$
\mathbf{B}_{j}^{S-T}=\left[\begin{array}{ll}
\mathbf{B}_{s p h} & \mathbf{B}_{\text {tran }}
\end{array}\right]
$$

where $\mathbf{B}_{s p h}=\left[\begin{array}{ll}\widetilde{\mathbf{r}}_{2} & \mathbf{I}\end{array}\right]^{T} \mathbf{A}_{1} \mathbf{C}_{12 S-T} 2 \mathbf{E}_{j}$ is the velocity transformation for the spherical joint, and $\mathbf{B}_{\text {tran }}=\left[\begin{array}{ll}\mathbf{g}_{i j} & \mathbf{0}\end{array}\right]^{T}$ is the velocity transformation vector for the translational joint, where $\mathbf{g}_{i j}=\mathbf{A}_{1} \mathbf{C}_{12 S-T} \mathbf{A}_{s p h}^{\prime \prime}\left[\begin{array}{lll}0 & 0 & 1\end{array}\right]^{T}$ and the transformation matrix $\mathbf{A}_{2}$ can be written as $\mathbf{A}_{2}=\mathbf{A}_{1} \mathbf{C}_{12 S-T} \mathbf{A}_{s p h}^{\prime \prime}$.

When a given initial estimate does not satisfy the constraint equations $\Phi$, the set of linear equations $\Phi_{\mathbf{q}} \delta \mathbf{q}=-\Phi$ are solved simultaneously. The variables $\mathbf{q}$ are updated until the constraints are satisfied. In this example, the linear equations are written as

$$
\left[\begin{array}{cc}
\Phi_{\mathbf{q}_{S-T}^{R-S}}^{R} & \mathbf{0} \\
\Phi_{\mathbf{q}_{S-T}^{T-D}}^{T-} & \Phi_{q_{3}}^{T-D}
\end{array}\right]\left[\begin{array}{c}
\delta \mathbf{q}_{S-T} \\
\delta q_{3}
\end{array}\right]=\left[\begin{array}{c}
-\Phi^{R-S} \\
-\Phi^{T-D}
\end{array}\right]
$$

where the $(2 \times 5)$ Jacobian matrix $\Phi_{\mathbf{q}_{S-T}}^{R-S}$ and the $(1 \times 5)$ vector $\Phi_{\mathbf{q}_{S-T}}^{T-D}$, in joint coordinate space, are transformed from the Cartesian space $\Phi_{\hat{\mathbf{z}}_{j}}$ (where $\Phi_{\hat{\mathbf{z}}_{j}}^{R-S}$ has dimension $2 \times 6$ and $\Phi_{\hat{\mathbf{z}}_{j}}^{T-D}$ has dimension $1 \times 6$ ) by using the velocity transformation matrix $\mathbf{B}$, such that $\Phi_{\mathbf{q}_{S-T}}^{R-S}=\Phi_{\hat{\mathbf{z}}_{j}}^{R-S} \mathbf{B}_{j}^{S-T}$ and $\Phi_{\mathbf{q}_{S-T}}^{T-D}=\Phi_{\hat{\mathbf{z}}_{j}}^{T-D} \mathbf{B}_{j}^{S-T}$. Initial estimates of the position of the chassis is given as $\left[\begin{array}{lll}x & y & z\end{array}\right]=\left[\begin{array}{lll}0.0 & 0.0 & 0.55\end{array}\right]^{T}$ and the orientation in terms of Euler parameters as $\left[\begin{array}{lll}e_{1} & e_{2} & e_{3}\end{array}\right]=\left[\begin{array}{lll}0.0 & 0.0 & 0.0\end{array}\right]^{T}$.

Initial estimates of the joint position vectors in body reference frame are entered into column 2 of Table 2. Estimates of transformation matrices $\mathbf{C}_{i j}$ from Joint definition frames to body reference frames are computed as

$$
\mathbf{C}_{12 R-s}=\left[\begin{array}{ccc}
0.000256112 & -0.99961 & -0.0261336 \\
0.00979628 & 0.0261336 & -0.99961 \\
0.999904 & 0 & 0.0098001
\end{array}\right] \mathbf{C}_{21 R-s}=\mathbf{C}_{12 S-T}=\mathbf{C}_{21 T-D}=\left[\begin{array}{ccc}
0 & -1 & 0 \\
1 & 0 & 0 \\
0 & 0 & 1
\end{array}\right] \text { and } \mathbf{C}_{12 T-D}=\left[\begin{array}{ccc}
0 & 0 & 1 \\
1 & 0 & 0 \\
0 & 1 & 0
\end{array}\right]
$$

and the corresponding Euler parameters $\mathbf{P}_{i j}$ are computed as $\mathbf{P}_{\text {rR- }-\mathrm{s}}=\left[\begin{array}{lllll}0.508967 & 0.491 & -0.50398 & 0.495811\end{array}\right]^{\mathrm{T}}$

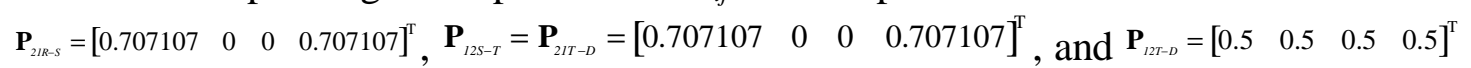
and estimates of the generalized coordinates are $\mathbf{q}_{1}=\left[\begin{array}{llll}1 / \sqrt{2} & 0 & 0 & -1 / \sqrt{2}\end{array}\right]^{T}, \quad q_{2}=-0.425$, and $q_{3}=0.305$. The initial estimates do not satisfy the constraint equations $\Phi$. The NewtonRaphson iteration method is used to update the generalized coordinates $\mathbf{q}$. The algorithm converges within 2 iterations within a tolerance of $10^{-5}$ and the set of new generalized coordinates are computed as: $\mathbf{p}_{1}=\left[\begin{array}{llll}0.6979 & -0.0320 & -0.0529 & -0.7137\end{array}\right]^{T}, q_{2}=-0.4337$, and $q_{3}=-0.2760$. Once the configuration changes, the constraint equations are violated. There are five possible methods to compensate the constraint violations. Those methods are:

(1) Change the generalized coordinates $\mathbf{q}=\left[\begin{array}{lll}\mathbf{p}_{1}^{T} & q_{2} & q_{3}\end{array}\right]$ by iteratively solving

$$
\Phi_{\hat{\mathbf{z}}_{2}} \mathbf{B}_{2}^{S-T} \delta \mathbf{q}_{S-T}+\Phi_{q_{3}} \delta q_{3}=-\Phi
$$

For a change in design parameters of the $\mathrm{S}-\mathrm{T}$ joint position vector $\mathbf{s}_{12 S-T}$ from $\left[\begin{array}{lll}0.5011 & 1.1162 & 0.1998\end{array}\right]^{T}$ to $\left[\begin{array}{lll}0.5 & 1.0 & 0\end{array}\right]^{T}$, only allowing the generalized joint variable to change using Eq. (60) while keeping invariant joint position vectors, the Newton-Raphson 
iteration method is used to compute an assembled configuration which is entered into column 3 of Table 1.

A designer, however, may opt to have constant generalized coordinates but allow the automatic computation of new joint position vectors, hence, changing the dimensions of links. Therefore, the second method can be used.

(2) Change the joint position vectors $\mathbf{S}_{i j}^{\prime}$ by iteratively solving

$$
\Phi_{\hat{\mathbf{z}}_{2}} \mathbf{M}_{1} \delta \mathbf{s}_{12 S-T}^{\prime}+\Phi_{\mathbf{s}_{12 R-S}^{\prime}} \delta \mathbf{s}_{12 R-S}^{\prime}+\Phi_{\mathbf{s}_{21 R-S}^{\prime}} \delta \mathbf{s}_{21 R-S}^{\prime}+\Phi_{\mathbf{s}_{12 T-D}^{\prime}} \delta \mathbf{s}_{12 T-D}^{\prime}+\Phi_{s_{21 T-D}^{\prime}} \delta \mathbf{s}_{21 T-D}^{\prime}=-\Phi
$$

Simulation results due to changing $\mathbf{s}_{12 S-T}$ using Eq. (61) are entered into column 4 of Table 1. A schematic illustrating these two methods is shown in Fig. 6. Method 1 has resulted in changing only the generalized coordinates without changing the joint position vectors. Method 2, illustrated in Fig. 6b, has changed the joint position vectors and therefore, has changed the structural configuration.
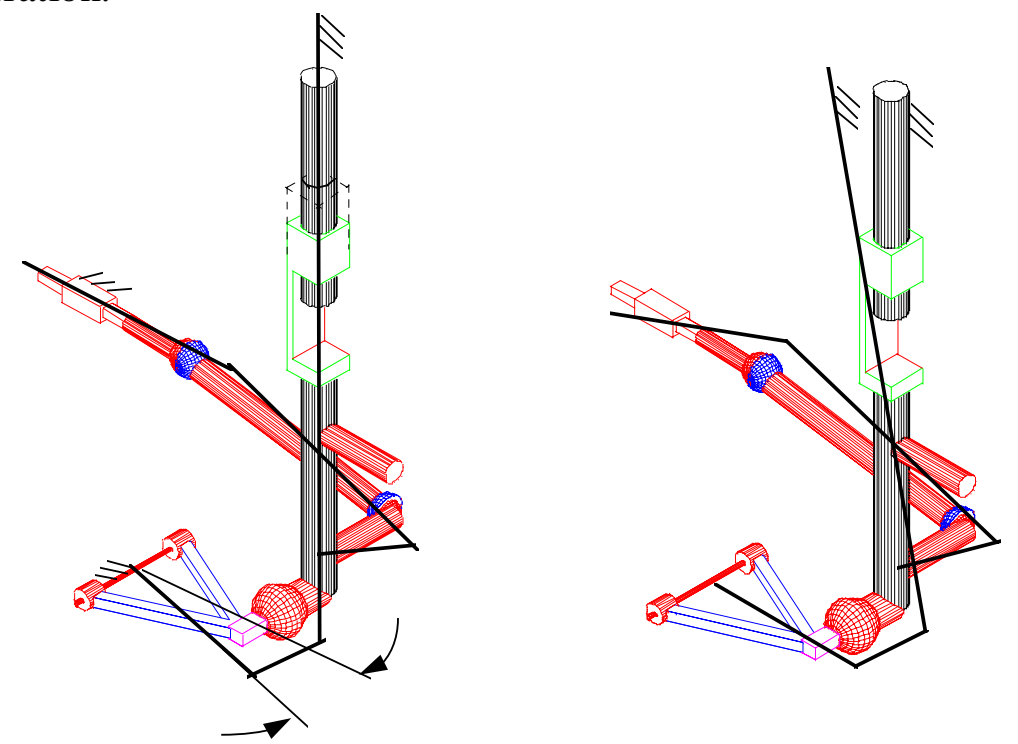

Fig. 6 (a) The mechanism after allowing a change in the generalized variables and

(b) the mechanism after a change in the joint position vectors

Simulation results due to allowing changes in both the joint position vectors and the generalized variables can be computed using the following method.

(3) Change the joint position vectors $\mathbf{s}_{i j}$ and generalized coordinates $\mathbf{q}$ by iteratively solving

$$
\begin{aligned}
\Phi_{\hat{\mathbf{z}}_{2}} \mathbf{B}_{2}^{S-T} \delta \mathbf{q}_{S-T}+ & \Phi_{q_{3}} \delta q_{3}+\Phi_{\hat{\mathbf{z}}_{2}} \mathbf{M}_{1} \delta \mathbf{s}_{12 S-T}^{\prime}+\Phi_{\mathbf{s}_{21 R-S}^{\prime}} \delta \mathbf{s}_{21 R-S}^{\prime}+\Phi_{\mathbf{s}_{21 R-S}^{\prime}} \delta \mathbf{s}_{21 R-S}^{\prime} \\
& +\Phi_{\mathbf{s}_{12 T-D}^{\prime}} \delta \mathbf{s}_{12 T-D}^{\prime}+\Phi_{\mathbf{s}_{21 T-D}^{\prime}} \delta \mathbf{s}_{21 T-D}^{\prime}=-\Phi
\end{aligned}
$$

simulation results using Eq. (62) are presented in column 6 of table 1.

(4) Change the joint position vectors $\mathbf{s}_{i j}^{\prime}$, lower control arm length $l_{R-S}$ and tie rod length $l_{T-D}$ by iteratively solving

$\Phi_{\hat{\mathbf{z}}_{2}} \mathbf{M}_{1} \delta \mathbf{s}_{12 S-T}^{\prime}+\Phi_{\mathbf{s}_{12 R-S}^{\prime}} \delta \mathbf{s}_{12 R-S}^{\prime}+\Phi_{\mathbf{s}_{21 R-S}^{\prime}} \delta \mathbf{s}_{21 R-S}^{\prime}+\Phi_{\mathbf{s}_{12 T-D}^{\prime}} \delta \mathbf{s}_{12 T-D}^{\prime}+\Phi_{\mathbf{s}_{21 T-D}^{\prime}} \delta \mathbf{s}_{21 T-D}^{\prime}+\Phi_{l} \delta l=-\Phi$

Simulation results using Eq. (63) are entered in column 5 of table 1.

Change the joint position vectors $\mathbf{s}_{i j}^{\prime}$, generalized coordinates $\mathbf{q}$, lower control arm length $l_{R-S}$, and tie rod length $l_{T-D}$ by iteratively solving 


$$
\begin{gathered}
\Phi_{\hat{\mathbf{z}}_{2}} \mathbf{B}_{2}^{S-T} \delta \mathbf{q}_{S-T}+\Phi_{q_{3}} \delta q_{3}+\Phi_{\hat{\mathbf{z}}_{2}} \mathbf{M}_{1} \delta \mathbf{s}_{1_{12 S-T}}^{\prime}+\Phi_{\mathbf{s}_{21 R-S}^{\prime}} \delta \mathbf{s}_{21 R-S}^{\prime}+\Phi_{\mathbf{s}_{21 R-S}} \delta \mathbf{s}_{21 R-S}^{\prime} \\
+\Phi_{\mathbf{s}_{12 T-D}} \delta \mathbf{s}_{12 T-D}^{\prime}+\Phi_{\mathbf{s}_{21 T-D}^{\prime}} \delta \mathbf{s}_{21 T-D}^{\prime}+\Phi_{l} \delta l=-\Phi
\end{gathered}
$$

Simulation results for computing an assembled configuration using Eq. (64) are presented in column 7 of Table 1. It is noted that only a few number of iterations is required to converge to an assembled mechanism.

\begin{tabular}{|c|c|c|c|c|c|c|}
\hline $\begin{array}{c}1 \\
\text { variables }\end{array}$ & $\begin{array}{c}2 \text { after } \\
\text { initial } \\
\text { assembly }\end{array}$ & $\begin{array}{l}3 \\
\mathbf{q}\end{array}$ & $\begin{array}{c}4 \\
\vdots \\
\mathbf{s}_{i j}\end{array}$ & $\mathbf{s}_{i j},_{R-S}^{5} l_{T-D}$ & $\begin{array}{c}6 \\
\mathbf{s}_{i j}, \mathbf{q}\end{array}$ & $\begin{array}{c}\mathbf{s}_{i j}^{\prime}, \mathbf{q} \\
l_{R-S}, l_{T-D}\end{array}$ \\
\hline $\mathbf{q}_{l}$ & {$\left[\begin{array}{c}0.6980 \\
-0.0320 \\
-0.0529 \\
-0.7137\end{array}\right]$} & {$\left[\begin{array}{c}0.6870 \\
0.0784 \\
-0.0912 \\
-0.7166\end{array}\right]$} & & & {$\left[\begin{array}{c}0.6995 \\
0.0199 \\
-0.0666 \\
-0.7112\end{array}\right]$} & {$\left[\begin{array}{c}0.6993 \\
0.0166 \\
-0.0686 \\
-0.7114\end{array}\right]$} \\
\hline $\mathrm{q}_{2}$ & -0.4337 & -0.3638 & & & $\begin{array}{l}-0.4061 \\
\end{array}$ & $\begin{array}{l}-0.4106 \\
\end{array}$ \\
\hline $\mathrm{q}_{3}$ & 0.2760 & 0.2349 & & & 0.2799 & 0.2806 \\
\hline $\mathbf{S}_{I 2 S-T}$ & {$\left[\begin{array}{l}0.5011 \\
1.1162 \\
0.1998\end{array}\right]$} & {$\left[\begin{array}{c}0.5 \\
1.0 \\
0\end{array}\right]$} & {$\left[\begin{array}{c}0.5 \\
1.0 \\
0\end{array}\right]$} & {$\left[\begin{array}{c}0.5 \\
1.0 \\
0\end{array}\right]$} & {$\left[\begin{array}{c}0.5 \\
1.0 \\
0\end{array}\right]$} & {$\left[\begin{array}{c}0.5 \\
1.0 \\
0\end{array}\right]$} \\
\hline $\mathbf{S}_{I 2 R-S}$ & {$\left[\begin{array}{c}0.33 \\
1.1735 \\
-0.3923\end{array}\right]$} & & {$\left[\begin{array}{c}0.3647 \\
1.1148 \\
-0.4365\end{array}\right]$} & {$\left[\begin{array}{c}0.3551 \\
1.1152 \\
-0.4246\end{array}\right]$} & {$\left[\begin{array}{c}0.3485 \\
1.1419 \\
-0.4162\end{array}\right]$} & {$\left[\begin{array}{c}0.3449 \\
1.1421 \\
-0.4116\end{array}\right]$} \\
\hline $\mathbf{S}_{2 l R-S}$ & {$\left[\begin{array}{c}-0.0507 \\
0.0321 \\
-0.2044\end{array}\right]$} & & {$\left[\begin{array}{c}-0.0813 \\
0.0914 \\
-0.1580\end{array}\right]$} & {$\left[\begin{array}{c}-0.0731 \\
0.0909 \\
-0.1709\end{array}\right]$} & {$\left[\begin{array}{c}-0.0674 \\
0.0649 \\
-0.1797\end{array}\right]$} & {$\left[\begin{array}{c}-0.0642 \\
0.0644 \\
-0.1846\end{array}\right]$} \\
\hline $\mathbf{S}_{2 I T-D}$ & {$\left[\begin{array}{c}0 \\
1.2675 \\
-0.3748\end{array}\right]$} & & {$\left[\begin{array}{c}0.0298 \\
1.2597 \\
-0.3931\end{array}\right]$} & {$\left[\begin{array}{c}0.0210 \\
1.2620 \\
-0.3876\end{array}\right]$} & {$\left[\begin{array}{c}0.0039 \\
1.2663 \\
-0.3772\end{array}\right]$} & {$\left[\begin{array}{c}0.0046 \\
1.2663 \\
-0.3776\end{array}\right]$} \\
\hline $\mathbf{S}_{2 I T-D}$ & {$\left[\begin{array}{c}0.07 \\
0.155 \\
-0.186\end{array}\right]$} & & {$\left[\begin{array}{c}0.0424 \\
0.1627 \\
-0.1645\end{array}\right]$} & {$\left[\begin{array}{c}0.0506 \\
0.1604 \\
-0.1709\end{array}\right]$} & {$\left[\begin{array}{c}0.0665 \\
0.1561 \\
-0.1831\end{array}\right]$} & {$\left[\begin{array}{c}0.0658 \\
0.1562 \\
-0.1827\end{array}\right]$} \\
\hline$l_{R-S}$ & 0.203 & & & 0.2326 & & 0.2208 \\
\hline$l_{T-D}$ & 0.3742 & & & 0.3955 & & 0.3787 \\
\hline $\mathbf{P}_{12 S-T}$ & {$\left[\begin{array}{c}0.7071 \\
0 \\
0 \\
0.7071\end{array}\right]$} & & & & & \\
\hline $\mathbf{P}_{I 2 R-S}$ & {$\left[\begin{array}{c}0.5090 \\
0.491 \\
-0.5040 \\
0.4958\end{array}\right]$} & & & & & \\
\hline $\mathbf{P}_{I 2 T-D}$ & {$\left[\begin{array}{l}0.5 \\
0.5 \\
0.5 \\
0.5\end{array}\right]$} & & & & & \\
\hline$\Phi$ norm & 0.000672544 & 0.00015806 & 0.000348392 & 0.000564039 & $1.85401 \mathrm{e}-05$ & 0.000647021 \\
\hline Iterations & 2 & 3 & 3 & 2 & 3 & 2 \\
\hline
\end{tabular}

Table 1 Simulation results due to a change in $s_{12 S-T}^{\prime}$

\section{Conclusions}

An analytical formulation for studying kinematic design propagations for planar and spatial mechanisms is presented. The formulation presented in this paper and illustrated by a numerical example demonstrates the validity of a general purpose formulation and experimental computer code for the computation of an assembled mechanism due to a change in design parameters. 
A mechanism was shown to be modeled using graph theory and closed loops were converted into a tree structure using a cut-joint constraint formulation. It was shown that this formulation can be derived with respect to design parameters to obtain kinematic design propagations. Basic constraints were derived allowing for the variation of joint position vectors and orientation matrices written in terms of Euler parameters. A designer may use this experimental code to study different scenarios or test a new design.

It was shown that the Jacobian matrix in Cartesian space can be transformed to Joint coordinate space. The use of relative generalized coordinates in a recursive formulation has simplified obtaining the differential variations. It was shown that starting from an initial configuration that satisfies the independent constraints, an iterative technique can be used to compute the resulting variations. The method has showed to be applicable for a variety of design parameters.

The ultimate goal of this research is to obtain an analogous formulation for the variational characteristics of dynamics parameters during a design cycle. It will be advantageous to a designer, a kinematician, or a dynamicist to obtain relative velocities, accelerations and forces due to a change in the design.

\section{Acknowledgments}

This research was funded by the US Army Tank Automotive Research Center (TACOM) through the Automotive Research Center (Department of Defense contract number DAAE0794-C-R094) and the National Science Foundation (NSF) contract number ECS-9422722. The authors extend their thanks to Professor E. J. Haug for his insightful comments.

\section{References}

Alder, R. E. and Ishii, K., 1989, "DAISIE: Designer's aid for simultaneous engineering," Computers in Engineering Conf. and Expo. Vol. 1, pp. 19-26.

Bae, D. S. and Haug, E. J., 1987, “A Recursive formulation for constrainted mechanical system dynamics, Part I-Open Loop systems, and part II-Closed Loop systems," Mechanics of Structures and Machines, Vol. 15, No. 3 and No. 4.

Bae, D. S., Hwang, R. S., and Haug, E. J., 1988, "Recursive formulation for real-time dynamic simulation," Techanical Report D-2, Center for Simulation and Design Optimization, the University of Iowa, Iowa City, IA.

Bowen, J. and Bahler, D., 1992, "Frames, quantification, perspectives, and negotiation in constraint networks for life-cycle engineering," Artificial Intelligence. in Engineering Vol. 7 , pp. 199-226.

Burke, E. K., Elliman, D. G., and Heard, M. I., 1994, "XCODAMS: an engineering design system based on constraint propagation," Applications of Artificial Intelligence in Engineering, pp. 601-608.

Cutkosky, M. R. and Tenenbaum, J. M., 1990, "Toward a computational framework for concurrent engineering," Electronics Society-IECON, Pacific Grove, CA, pp. 700-706.

DADS User's Manual, Computer Aided Design Software Inc. (CADSI), P.O.Box 203, Oakdale, Iowa, 52319.

Fisher, D. C. and Nguyen, T. D., 1989, "Design and analysis aid for evaluating aircraft structures," Proc. 3rd Int. Conf. on Industrial and Engineering Applications of Artificial Intelligence and Expert Systems IEA/AIE, Charleston, pp. 469-472. 
Fohn, S. M . Greef, A. R. Young, R. E. and O'Grady, P. J., 1994, “Constraint system shell to support engineering approaches to concurrent engineering," Artificial Intelligence in Engineering Vol. 9, No. 1, pp. 1-17.

Gopalakrishnan, B. and Pandiarajan, V., 1990, "Product design for manufacturing: the use of knowledge based systems in concurrent engineering," IEEE Int Conf. on Systems, man and Cybernetics, Los Angeles, CA, pp. 566-568.

Haug, E. J., 1989, Computer Aided Kinematics and Dynamics of Mechanical Systems Vol I Allyn \& Bacon.

Hwang, R. S. and Haug, E. J., 1988, "A recursive multibody dynamics formulation for parallel computation," Technical Report R-13, Center for Simulation and Design Optimization, the University of Iowa, Iowa City, IA.

Hwang, R. S., Bae, D. S., Kuhl, J. G., and Haug, E. J., 1988, "Parallel processing for realtime dynamic system simulation," Journal of Mechanisms, Transmissions, and Automation in Design.

Imamura, S., 1994, "Development of constraint based object oriented language for engineering design-on the parametric design support," Journal of the Japan Society for Precision Engineering Vol. 60, No. 9, pp. 1242-1246.

Kolb, M. A. and Bailey, M. W., 1993, "FRODO: constraint-based object modeling for preliminary design," Proceedings of the 19th Annual ASME Design Automation Conference, Albuquerque, NM, Vol. 65, pp. 307-318.

Serrano, D. and Gossard, D., 1988, "Constraint management," MCAE' Artificial Intelligence in Engineering Design (J. S. Gero, editor), Elsevier, Amsterdam, pp. 217-239.

Silverstein, E., Kaminski, J. S., Farenci, R., and Kumaran, A., 1990, "A knowledge acquisition tool for design engineering applications," Proc. 3rd Int. Conf. on Industrial and Engineering Applications of Artificial Intelligence and Expert Systems IEA/AIE, Charleston, pp. $1167-1172$

Tak, T. and Kim, S. S., 1990, "Design sensitivity analysis of multibody dynamic systems," Technical Report R-88, Center for Simulation and Design Optimization, the University of Iowa, Iowa City, IA.

Tsai, F. F., 1989, "Automatic method for high speed simulation of multibody dynamic systems," PhD Dissertation, The University of Iowa, Iowa City, IA.

Wittenburg, J., 1977, Dynamics of systems of rigid bodies, (B G Teubner), Stuttgart, 1977. 